\title{
Recent CMS Results on Diffraction
}

\author{
Roland Benoit on behalf of the CMS Collaboration \\ $D E S Y$ - CMS \\ Notkestrasse 85, 22603 Hamburg \\ Germany
}

\begin{abstract}
Recent CMS results on diffraction are presented. These include the measurements of the soft diffractive cross sections, of the forward rapidity gap cross section, of the diffractive dijet cross section, the measurement of a large rapidity gap in $W$ and $Z$ boson events and the measurement of the pseudorapidity distribution of charged particles in a single diffractive enhanced sample. This last measurement is the first common result of the CMS and TOTEM collaborations. Some prospects of common CMS-TOTEM data taking are also discussed.
\end{abstract}

Keywords: Quantum Chromodynamics, Diffractive Processes, Gap Survival Probability.

PACS: 12.38.-t, 12.38.Qk.

\section{Diffraction}

Diffractive reactions represent a sizable fraction of the total inelastic cross section $\sigma_{\text {tot }}$ in hadronhadron scattering, of the order of $25 \%$ of $\sigma_{t o t}[1]$. These reactions are characterized by a color neutral $t$-channel exchange carrying the quantum numbers of the vacuum, where $t$ is the fourmomentum squared exchanged in the collision [2]. In proton-proton $(p p)$ collisions, single diffractive (SD) dissociation $p p \rightarrow p X$ corresponds to the process where one of the protons emerges intact in the final state, while the other is scattered into a low-mass system $X$. In double diffractive (DD) dissociation $p p \rightarrow X Y$, both of the protons are scattered into low-mass states $X$ and $Y$, whereas in central diffractive (CD) dissociation, $p p \rightarrow p X p$, both protons emerge intact from the collision, with a low-mass system $X$ produced centrally. In all cases, the energy of the outgoing protons or states $X, Y$ is approximately equal to that of the incoming protons, to within a few percent. Diffractive events are characterized by the presence of at least one large rapidity region $\Delta y$ devoid of hadronic activity [3], the so-called large rapidity gap (LRG), where the rapidity $y$ is defined as $y=\ln \left[\left(E+p_{Z}\right) /\left(E-p_{Z}\right)\right], E$ and $p_{Z}$ being the energy and longitudinal momentum of the final state particle, respectively. The pseudorapidity $\eta=-\ln [\operatorname{tg}(\theta / 2)]$, with $\theta$ the polar angle of the particle, is often used experimentally instead of rapidity. Both variables are equal in the limit of a massless particle.

Diffractive hadron-hadron collisions can be described in the framework of Regge theory $[2,4]$. In this framework, the $t$-channel process is governed by the exchange of resonances sitting on a so-called Regge trajectory $\alpha(t)=\alpha+\alpha^{\prime} t$. A resonance of mass $m$ sitting on the trajectory at $t=m^{2}$ has an angular momentum of value $\alpha\left(m^{2}\right)$, and a Regge trajectory therefore interpolates between resonances of increasing angular momentum. The contribution of a trajectory to the total inelastic cross section is given by $\sigma_{t o t}(s) \propto s^{\alpha-1}$, where $s$ is the centre-of-mass energy 
squared of the collision and $\alpha$ the intercept of the trajectory. In the Regge framework, diffraction is characterized by the exchange of a specific trajectory, the so-called Pomeron trajectory, which has the quantum numbers of the vacuum and an intercept $\alpha$ larger than one.

Hard diffractive electron-proton (ep) collisions can be described by perturbative Quantum Chromodynamics (QCD) in the framework of collinear factorization [5, 6]. In this framework, the cross section of a hard diffractive process is given by the convolution of a parton-level cross section, which is process-dependent, with the diffractive parton distribution functions (dPDFs), which are process-independent. The evolution of the dPDFs with the four-momentum squared exchanged at the proton vertex, $Q^{2}$, is governed by the DGLAP evolution equations [7-10]. In the parton model, these functions can be interpreted at leading-order as conditional probabilities to find a parton in the proton, carrying a fraction $x$ of its longitudinal momentum, at a given value of $Q^{2}$, under the assumption that a fast leading proton is present in the final state. Hard diffractive ep collisions have been studied extensively at the ep HERA collider through the measurement of both inclusive and exclusive processes [11-17]. The dPDFs have been determined by the HERA experiments $[11,12]$ by means of QCD fits to inclusive diffractive deep inelastic scattering data.

Hard diffractive processes such as the diffractive production of jets [18-20], or $W$ and $Z$ bosons [21] have been studied at hadron colliders. The measurement of diffractive dijet production in $p \bar{p}$ collisions from the CDF collaboration at the Tevatron [18] was the first to show an important disagreement with the theoretical expectations based on the dPDFs determined from HERA data. The fraction of diffractive dijet events is a factor 3 to 10 smaller than the expectation, with the reduction becoming more important with increasing value of the struck parton momentum fraction. Collinear factorization can not be applied to diffractive hadron-hadron collisions $[22,23]$, because additional soft or semi-hard interactions between the spectator partons, so-called multiple parton interactions (MPI), can occur and produce hadronic activity that lowers the probability for the rapidity gap to form. With this picture in mind, the reduction factor is often referred to as the rapidity gap survival probability $S^{2}$. Various models have been investigated to determine the expected behaviour of $S^{2}$ at the LHC centre-of-mass energy $\sqrt{s}=7 \mathrm{TeV}$. These models mainly differ on the type of the Pomeron amplitude describing the soft re-scatterings at the origin of the rapidity gap suppression [24-27]. Most of the theoretical calculations predict a value of $S^{2} \simeq 0.05$ for hard diffractive processes at LHC energies, but values as low as 0.004 and as high as 0.23 have been proposed [28].

Diffractive factorization breaking is intimately related to multiple scatterings in hadronhadron collisions. If one assumes that re-scatterings are independent of each other, the distribution of the number of re-scatterings at an impact parameter $b$ can be modelled by the Poisson statistics. Within this simple model, the gap survival probability is directly related to the mean number of re-scatterings at an impact parameter $b, \mu(b)$, through the relation [29]:

$$
S^{2}=\frac{\int d b \frac{d \sigma(b)}{d b} e^{-\mu(b)}}{\int d b \frac{d \sigma(b)}{d b}}
$$

where $\sigma(b)$ is the cross section for the hard diffractive process as a function of $b$. 


\section{Diffraction in CMS}

The study of diffraction at the LHC is of interest in several aspects. As diffractive reactions represent a sizeable fraction of the total proton-proton cross section, their contribution to the Underlying Event (UE), which denotes all the particles produced in a hard $p p$ scattering apart from the hard scattering system itself, is substantial. The CMS potential to achieve precise Standard Model measurements and to search for new physics is affected by the Underlying Event activity. Soft diffractive interactions, in which no hard scale is present, represent the bulk of the diffractive events. Given the importance of its contribution to the UE and the need to constrain its phenomenological description, the measurement of the soft diffractive cross section is therefore of primary importance.

The measurement of hard diffractive processes, in which the transverse momentum or the mass of the produced system defines the hard scale of the event, provides an important test of QCD and probes the low- $x$ structure of the proton, with $x$ the fractional momentum of the struck parton with respect to that of the incoming proton. In the low- $x$ region, the strong rise of the gluon density is expected to be tamed by recombination processes, leading to the saturation of the gluon density. The observation of this non-linear effect, which is not included in the DGLAP evolution equations [7-10], would shed light on the asymptotic high-energy behaviour of QCD.

Finally, the measurement of the rapidity gap survival probability, which quantifies the suppression of the diffractive cross section in $p p$ collisions with respect to ep scattering, is also of primary importance. The survival probability is poorly constrained, from both an experimental and a theoretical point of view, and its precise measurement would enable us to better understand the soft multiple parton interactions that occur in high-energy $p p$ collisions. MPI could fake or deteriorate signals for new physics, and a better knowledge of their dynamics would shed light on soft QCD, and would benefit precise measurements and searches for new physics as well.

During the LHC Run 1, diffractive processes have been mainly selected by requiring a large rapidity gap in the event, and all the CMS results presented in the following sections make use of the rapidity gap tagging to select diffractive events. The successful collaboration between the CMS and TOTEM experiments has recently enabled to complement the central CMS measurement with the scattered proton information measured with the TOTEM Roman pots in dedicated common runs during 2012 and 2013. Tagging the scattered proton(s) enables us to measure the four-momentum squared exchanged at the proton vertex, $t$, as well as the fractional momentum loss of the proton, $\xi$, when studying single diffractive dissociation $p p \rightarrow p X$ and central exclusive production (CEP) $p p \rightarrow p X p$. The study of these two processes with the CMS central detector only is affected by an irreducible proton dissociation background, which arises from events in which the scattered proton dissociates into a low-mass system $Y$ that escapes undetected. Tagging the scattered proton(s) makes it possible to significantly reduce the proton dissociation background when studying single diffractive dissociation and central exclusive production. In the case of CEP, tagging the scattered protons allows to reconstruct the mass of the centrally produced system with a resolution not achievable with the CMS detector only. The current ongoing analyses that make use of the proton tagging are dedicated to the study 
of single diffractive production of a dijet system, $J / \Psi$ or $D^{0}$ meson. While these analyses are using the information from both CMS and TOTEM, they suffer from low statistics and from the absence of dedicated triggers making use of the CMS central detector and TOTEM information.

Sections 3 and 4 give a short description of the CMS and TOTEM detectors, emphasizing on the subdetectors used in the diffractive analyses. The CMS results are presented in Sections 5 to 9 . These include the measurements of the soft diffractive cross sections [30], of the forward rapidity gap cross section [30], of the diffractive dijet cross section [31], the measurement of a large rapidity gap in $W$ and $Z$ boson events [32] and the measurement of the pseudorapidity distribution of charged particles in a single diffractive enhanced sample [33]. This last measurement is the first common result of the CMS and TOTEM collaborations. Some prospects of common CMS-TOTEM data taking are discussed in Sec.10, and a summary is given in Sec.11.

\section{The CMS detector}

A detailed description of the CMS detector can be found in Ref. [34]. The central feature of the apparatus is a superconducting solenoid of $6 \mathrm{~m}$ internal diameter, providing a $3.8 \mathrm{~T}$ axial field. Within the field volume are a silicon pixel and strip tracker, a crystal electromagnetic calorimeter (ECAL) and a brass/scintillator hadron calorimeter (HCAL). Muons are measured in gas-ionization detectors embedded in the steel flux return yoke. In addition to the barrel and endcap detectors, CMS has extensive forward calorimetry.

The CMS experiment uses a right-handed coordinate system, with the origin at the nominal interaction point (IP), the $x$-axis pointing to the center of the LHC, the $y$-axis pointing up (perpendicular to the plane of the LHC ring), and the $z$-axis along the anticlockwise-beam direction. The polar angle $\theta$ is measured from the positive $z$-axis and the azimuthal angle $\phi$ is measured in the $x-y$ plane.

The tracker measures charged particles within the pseudorapidity range $|\eta|<2.5$. It provides an impact parameter resolution of $\sim 15 \mu \mathrm{m}$ and a transverse momentum resolution of about $1.5 \%$ for $100 \mathrm{GeV} / c$ particles. ECAL and HCAL provide coverage in pseudorapidity up to $|\eta|<3$ in the barrel region and two endcap regions. The ECAL has an energy resolution of better than $0.5 \%$ above $100 \mathrm{GeV}$. The HCAL, when combined with the ECAL, measures jets with an energy resolution $\Delta E / E \approx 100 \% / \sqrt{E(\mathrm{GeV})} \oplus 5 \%$. The calorimeter cells are grouped in projective towers, of granularity $\Delta \eta \times \Delta \phi=0.087 \times 0.087$ at central rapidities and $0.175 \times 0.175$ at forward rapidities. The hadronic forward (HF) calorimeters cover the pseudorapidity region $2.9<|\eta|<5.2$. They consist of steel absorbers and embedded radiation-hard quartz fibres, which provide a fast collection of the Cherenkov light. Calorimeter cells are formed by grouping bundles of fibres. Clusters of these cells form a calorimeter tower. There are 13 towers in $|\eta|$, each with a size $\Delta \eta \approx 0.175$, except for the lowest- and highest- $|\eta|$ towers with $\Delta \eta \approx 0.1$ and $\Delta \eta \approx 0.3$, respectively. The azimuthal segmentation $\Delta \phi$ of all towers is $10^{\circ}$, except for the ones at highest- $|\eta|$, which have $\Delta \phi=20^{\circ}$. More forward angles, $-6.6<\eta<-5.2$, are covered by the CASTOR calorimeter [35], which is located only on the negative $z$-side of CMS, at $14.37 \mathrm{~m}$ from the IP. The calorimeter is segmented in $16 \phi$-sectors and $14 z$-modules, corresponding to 
a total of 224 cells. Each cell consists of quartz plates embedded in tungsten absorbers, with $45^{\circ}$ inclination with respect to the beam axis. Air core light guides provide a fast collection of the Cherenkov light. The first two modules, which have an absorber thickness half of that of the other modules, are used to detect electromagnetic showers. The full calorimeter has a depth of 10.5 nuclear interaction lengths.

\section{The TOTEM detector}

The TOTEM experiment $[36,37]$ is composed of three subdetectors: the Roman pots, and the $\mathrm{T} 1$ and T2 telescopes. The two T2 telescopes are placed symmetrically on each side of the IP at about $|z|=14 \mathrm{~m}$. They detect charged particles produced in the pseudorapidity region $5.3<|\eta|<6.5$, with full azimuthal acceptance. Each telescope consists of two half-arms, with each half-arm composed of 10 semicircular planes of triple-GEM (gas electron multiplier) chambers, arranged within a $40 \mathrm{~cm}$ space along the $z$-axis. Every chamber has a doublelayered readout board containing two columns of 256 concentric strips to measure the radial coordinate and a matrix of 1560 pads, each covering $\Delta \eta \times \Delta \phi=0.06 \times 0.018$, to measure the azimuthal coordinate. The radial and azimuthal coordinate resolutions are about $110 \mu \mathrm{m}$ and $1^{\circ}$, respectively [38]. Local angles of single tracks are reconstructed with an average resolution of $0.5 \mathrm{mrad}$, and the track pseudorapidity resolution for charged particles is better than 0.05 [39], once the track is identified as coming from the vertex.

\section{$5 \quad$ Soft diffractive cross sections}

The measurement of the SD and DD dissociation cross sections [30] is based on a sample of $p p$ collisions at $\sqrt{s}=7 \mathrm{TeV}$ selected online by a minimum-bias (MB) trigger. The data sample was collected when the LHC was operating in a low pileup scenario and corresponds to an integrated luminosity of $16.2 \mu \mathrm{b}^{-1}$ with an average number of inelastic $p p$ collisions per bunch crossing of 0.14 . This minimum-bias sample is dominated by nondiffractive (ND) events in which the production of the final state particles extends over the entire available phase-space. The presence of a rapidity gap in a ND event is due to statistical fluctuations in the production of the final state particles, and large rapidity gaps in ND events are therefore exponentially suppressed. In contrast, diffractive events are characterized by the presence of at least one nonexponentially suppressed LRG. The diffractive topologies defined experimentally according to the position of the LRG are presented in figure 1, where the open boxes represent the central CMS detector $(|\eta| \lesssim 4.7)$, the dotted open boxes the CASTOR calorimeter and the full boxes the final state systems.

The samples FG1 and FG2 (Forward Gap) correspond to diffractive events with a forward pseudorapidity gap reconstructed at the edge of the detector, on the positive (FG1) or negative (FG2) $\eta$-side. The CG sample (Central Gap) corresponds to diffractive events with a rapidity gap reconstructed in the detector around $\eta=0$. For the experimental topology FG1 (FG2), the forward rapidity gap is related to the variable $\eta_{\max }\left(\eta_{\min }\right)$ defined as the highest (lowest) $\eta$ of the particle candidate reconstructed in the central detector. For the CG topology, the pseudorapidity gap is expressed as $\Delta \eta^{0}=\eta_{\max }^{0}-\eta_{\min }^{0}$, with $\eta_{\max }^{0}\left(\eta_{\min }^{0}\right)$ the closest-to-zero $\eta$ 
value of the particle candidate reconstructed on the positive (negative) $\eta$-side of the detector. The detector-level distributions of $\eta_{\max }, \eta_{\min }$ and $\Delta \eta^{0}$ are presented in figure 2 and compared to the predictions of the Monte Carlo (MC) event generator PYTHIA8-MBR [40]. The ND contribution dominates the distributions at low values of the gap size, while the flattening of the exponential behaviour with increasing value of the gap is due to the contribution of the diffractive events. These dominate the distributions at low values of $\eta_{\max }$, high values of $\eta_{\min }$ or high values of $\Delta \eta^{0}$.

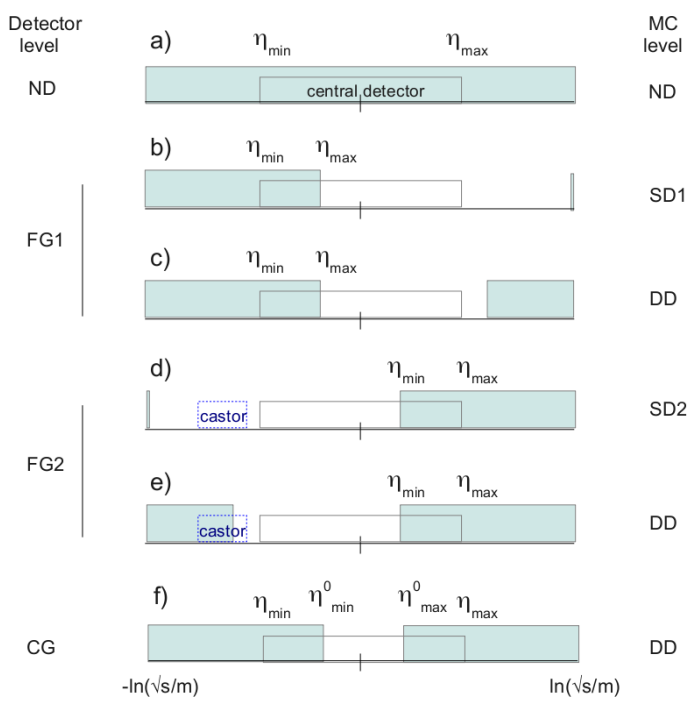

Figure 1: Diffractive topologies defined experimentally. Detector level: non diffractive events (ND), diffractive events with a forward pseudorapidity gap on the positive (FG1) or negative (FG2) $\eta$-side of the detector, or with a central gap (CG). MC level: ND events $p p \rightarrow X$, SD1 (SD2) events $p p \rightarrow X p(p p \rightarrow p X)$ and DD events $p p \rightarrow X Y$.
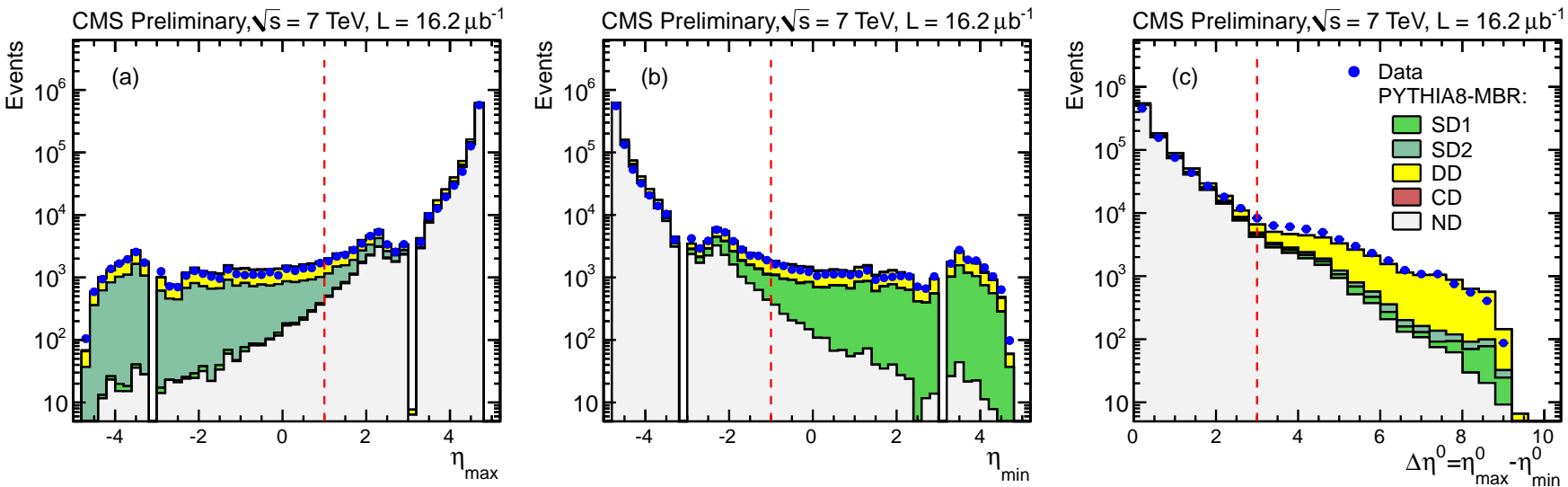

Figure 2: Detector-level distributions of $\eta_{\max }(\mathrm{a}), \eta_{\min }$ (b) and $\Delta \eta^{0}$ (c) for the minimum-bias sample. The data are compared to the predictions of the MC event generator PYTHIA8-MBR. Contributions are shown for each process separately. The dashed vertical lines represent the selections $\eta_{\max }<1, \eta_{\min }>-1$ and $\Delta \eta^{0}>3$, respectively. 
The diffractive-enhanced samples FG1 and FG2 are defined by the selections $\eta_{\max }<1$ and $\eta_{\min }>-1$, respectively. This condition corresponds to the presence of a forward LRG of at least 3.7 units in pseudorapidity. The diffractive-enhanced CG sample is defined by the selection $\Delta \eta^{0}>3$. This sample, for which both diffractive masses are required to be in the central detector, is dominated by ND and DD events. The diffractive-enhanced samples FG1 $\left(\eta_{\max }<1\right)$ and FG2 $\left(\eta_{\min }>-1\right)$ receive contributions from SD and DD processes in approximately equal amount. The DD contribution originates from events in which one of the dissociated systems has a low-mass and is produced outside of the acceptance of the central detector $(|\eta| \lesssim 4.7)$, as illustrated in the figure $1 \mathrm{c}$ ) and e). In the case of the FG1 sample, no detector is present on the positive $\eta$-side of CMS to measure a low-mass dissociated system that escapes detection in the central detector, and the FG1 sample is regarded as a control sample. For the FG2 sample, the CASTOR calorimeter can be used to tag the presence of a low-mass dissociated system in the pseudorapidity range $-6.6<\eta<-5.2$, to further divide the FG2 sample into a SD-enhanced subsample and a DD-enhanced subsample, according to the absence or the presence of activity in CASTOR, respectively.

The detector-level distributions of $\log _{10} \xi$ are presented in figure 3 for the entire FG2 sample (a), and for the subsamples without activity in CASTOR (b) and with activity in CASTOR (c). The FG2 sample is clearly separated into a SD-enhanced subsample (b) and a DD-enhanced subsample (c). The data are compared to predictions of the MC event generators PYTHIA8MBR and PYTHIA8 [41] with tune 4C [42]. The fractional momentum loss of the proton, $\xi$, is reconstructed from the energy $E^{i}$ and the longitudinal momentum $p_{z}^{i}$ of the particle candidates measured in the central detector, according to the relation $\xi=\sum\left(E^{i}-p_{z}^{i}\right) / \sqrt{s}$. The MC event generator PYTHIA8-MBR is found to give a better description of the data and is used to determine the diffractive cross sections.

The SD and DD differential cross sections as a function of $\log _{10} \xi$ are determined from the subsamples without activity in CASTOR and with activity in CASTOR, respectively. The correction for the detector acceptance and the subtraction of the background contributions are determined with the PYTHIA8-MBR MC event generator. The dominant background contribution to the SD cross section originates from DD events. For the DD cross section, the dominant background contribution originates from ND events. The cross sections are measured in the range $-5.5<\log _{10} \xi<-2.5$. For the DD cross section, the mass of the system $Y$ is limited to the region $0.5<\log _{10}\left(M_{Y} / \mathrm{GeV}\right)<1.1$ corresponding to the acceptance of the CASTOR calorimeter. The dominant sources of systematic uncertainties are the energy scale of the HF calorimeter and the modelling of hadronization and diffraction. The measured cross sections are presented in figure 4 and compared to predictions of the MC event generators PYTHIA8-MBR, PYTHIA8-4C and PYTHIA6 [43] with tune D6T [44]. The predictions from PYTHIA8MBR are shown for two values of the Pomeron intercept, $\alpha=1.08$ and $\alpha=1.104$. Both values describe the SD cross section within uncertainties, while the DD cross section is better described by the smaller value of the intercept. The predictions from PYTHIA8-4C and PYTHIA6D6T, based on the Schuler-Sjostrand model, give a good description of the DD cross section but fail to describe the falling behavior of the SD cross section. 

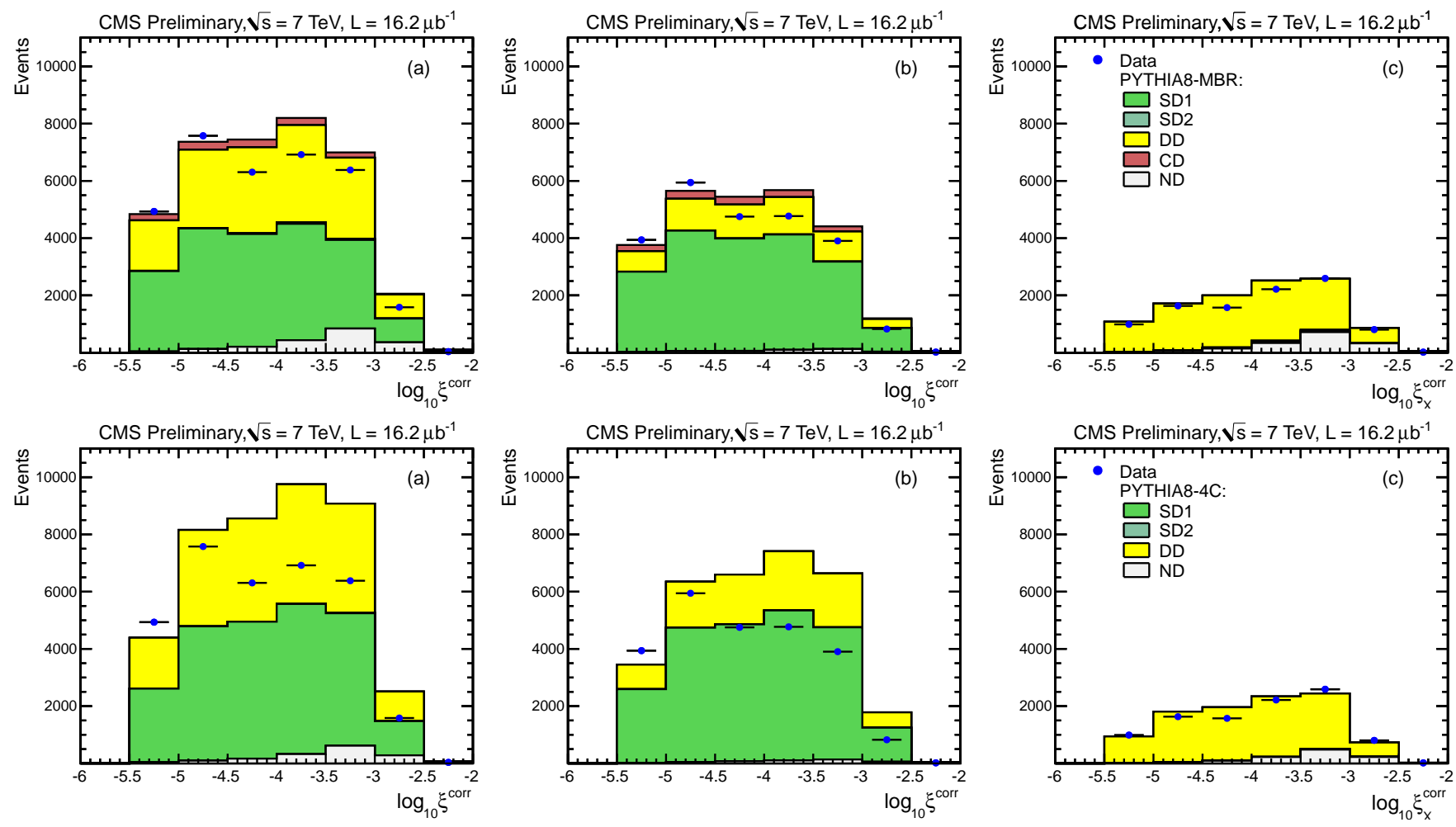

Figure 3: Detector-level distributions of the variable $\xi$ for the entire FG2 sample (a), and for the subsamples without activity in CASTOR (b) and with activity in CASTOR (c). The data are compared to predictions of the MC event generators PYTHIA8-MBR (top) and PYTHIA8-4C (bottom). Contributions are shown for each process separately.
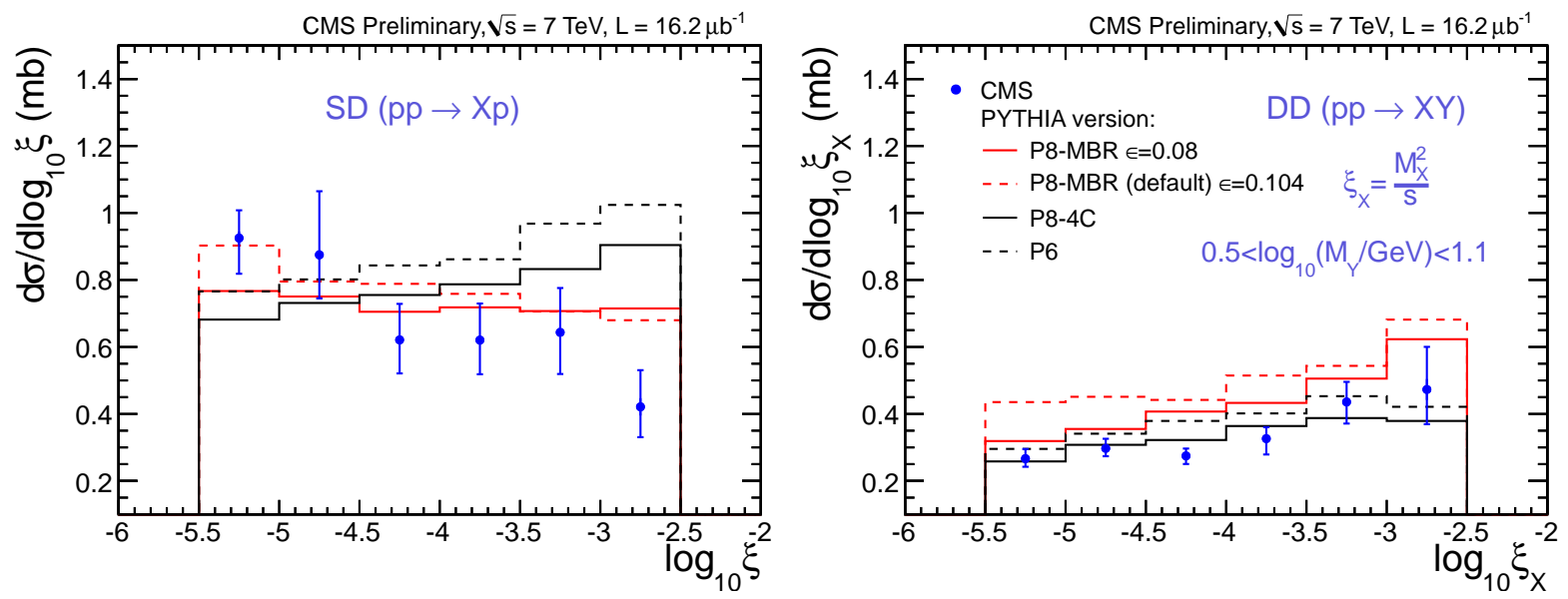

Figure 4: The SD and DD differential cross sections as a function of $\xi$. The measurements are compared to predictions of the MC event generators PYTHIA8-MBR, PYTHIA8-4C and PYTHIA6-D6T. The error bars represent the statistical and systematic uncertainties added in quadrature. 
The DD differential cross section as a function of the size of the pseudorapidity gap, $\Delta \eta$, is determined from the CG sample. The variable $\Delta \eta$ is defined as $\Delta \eta=-\log \xi$, with $\xi=M_{X}^{2} \cdot M_{Y}^{2} /\left(s \cdot m_{p}^{2}\right), M_{X}\left(M_{Y}\right)$ the invariant mass of the system $X(Y)$ and $m_{p}$ the proton mass. The cross section is measured in the range $\Delta \eta>3, M_{X}>10 \mathrm{GeV}$ and $M_{Y}>10 \mathrm{GeV}$. The correction for the detector acceptance and the subtraction of the background are determined with the PYTHIA8-MBR MC event generator. The dominant background contribution originates from ND events. The measured cross section is presented in figure 5 and compared to predictions of the MC event generators PYTHIA8-MBR, PYTHIA8-4C and PYTHIA6D6T. The predictions give a reasonable description of the data within uncertainties.

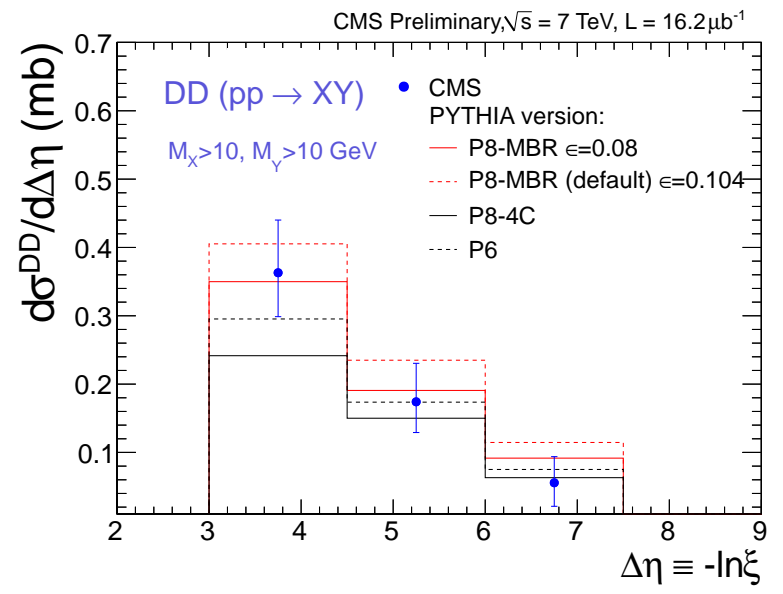

Figure 5: The DD differential cross section as a function of $\Delta \eta$. The measurement is compared to predictions of the MC event generators PYTHIA8-MBR, PYTHIA8-4C and PYTHIA6D6T. The error bars represent the statistical and systematic uncertainties added in quadrature.

The total SD cross section at $\sqrt{s}=7 \mathrm{TeV}$ integrated over the region $-5.5<\log _{10} \xi<-2.5$ is measured. A value of $4.27 \pm 0.04$ (stat.) ${ }_{-0.58}^{+0.65}$ (syst.) $\mathrm{mb}$ is extracted from the data. The total DD cross section integrated over the region $\Delta \eta>3, M_{X}>10 \mathrm{GeV}$ and $M_{Y}>10 \mathrm{GeV}$ is also measured. A value of $0.93 \pm 0.01$ (stat. $)_{-0.22}^{+0.26}$ (syst.) $\mathrm{mb}$ is extracted from the data.

\section{Forward rapidity gap cross section}

The measurement of the forward rapidity gap cross section [30] is based on a sample of $p p$ collisions at $\sqrt{s}=7 \mathrm{TeV}$ selected online by a MB trigger. The data sample corresponds to an integrated luminosity of $20.3 \mu \mathrm{b}^{-1}$ collected in a low pileup scenario with an average number of inelastic $p p$ collisions per bunch crossing of 0.007 . The largest forward rapidity gap, $\Delta \eta^{F}$, is measured in the acceptance of the central CMS detector $(|\eta|<4.7)$ and defined as $\Delta \eta^{F}=\max \left(4.7-\eta_{\max }, \eta_{\min }+4.7\right)$. The correction for the trigger efficiency and the subtraction of the beam induced background are determined in a data-driven way. The migration and detector acceptance corrections are evaluated with an iterative Bayesian unfolding technique [45]. The corrected results are defined for events with at least one final state particle of transverse momentum $p_{T}>200 \mathrm{MeV}$ in the region $|\eta|<4.7$. The dominant sources of systematic uncertainties are the energy scale of the HF calorimeter and the modelling of hadronization and diffraction. The forward rapidity gap cross section is presented in figure 6 and compared 

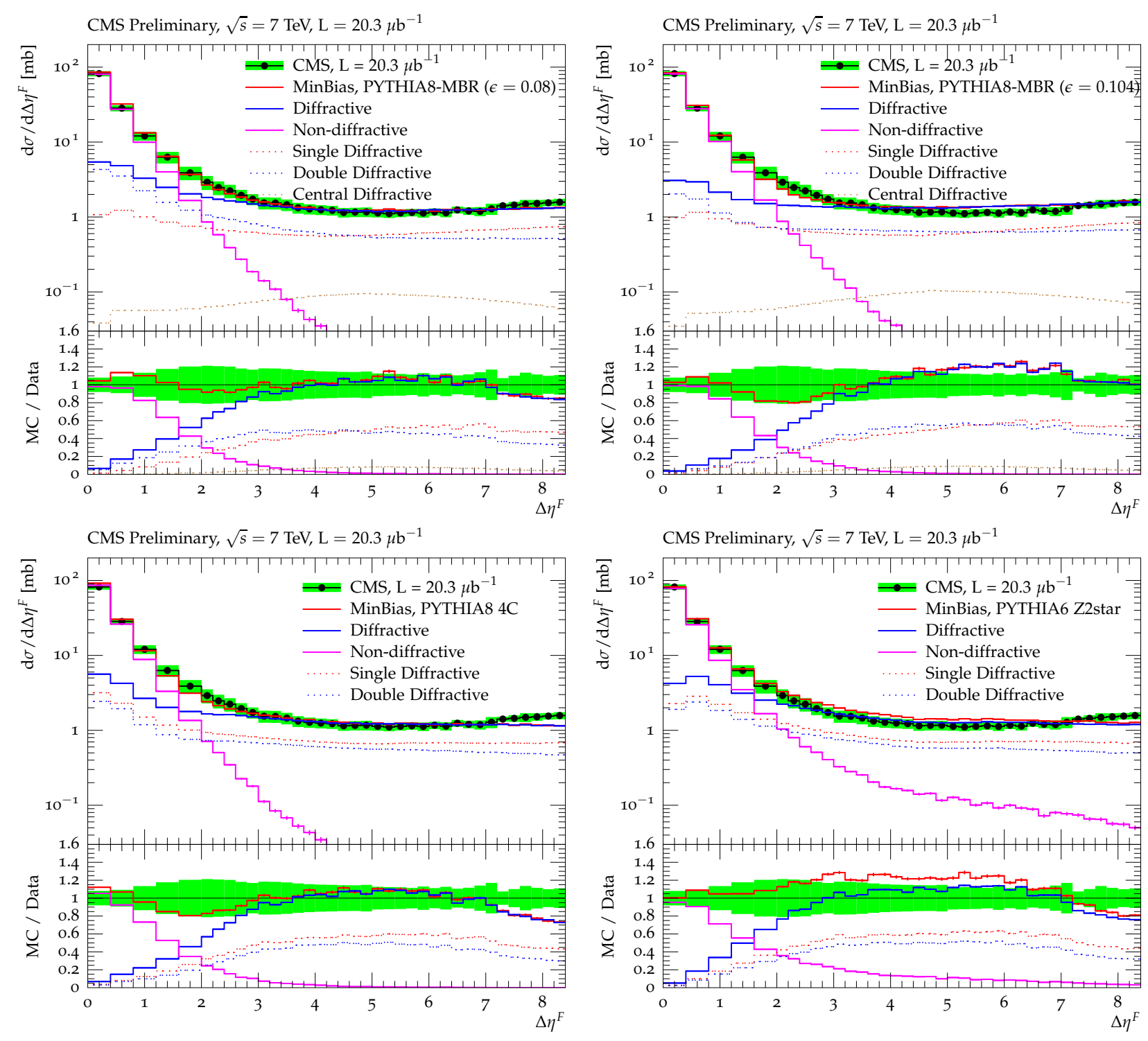

Figure 6: The forward rapidity gap cross section $d \sigma / d \Delta \eta^{F}$ for events with at least one final state particle of transverse momentum $p_{T}>200 \mathrm{MeV}$ in the region $|\eta|<4.7$. The measurement is compared to predictions of the MC event generators PYTHIA8-MBR, PYTHIA8-4C and PYTHIA6-Z2*. The bands represent the total systematic uncertainty.

to predictions of the MC event generators PYTHIA8-MBR, PYTHIA8-4C and PYTHIA6 with tune Z2* [46]. The predictions from PYTHIA8-MBR are shown for two values of the Pomeron intercept, $\alpha=1.08$ and $\alpha=1.104$. The bands represent the total systematic uncertainty of the order of $20 \%$. The minimum-bias sample is dominated by ND events in the region of small forward rapidity gap $\Delta \eta^{F}<3$. At larger $\Delta \eta^{F}$, the ND contribution is suppressed and the flattening of the exponential behaviour is due to the contribution of the diffractive events. Most of the predictions underestimate the cross section at large values of $\Delta \eta^{F}$. The prediction from PYTHIA8-MBR with a Pomeron intercept $\alpha=1.104$ gives the best description of the 
data at large values of $\Delta \eta^{F}$ but overestimates the cross section in the region of intermediate gap size. A comparison of the CMS and ATLAS [47] measurements is shown in figure 7. Except for the pseudorapidity coverage, which is slightly different between the CMS $(|\eta|<4.7)$ and ATLAS $(|\eta|<4.9)$ results, the hadron-level definitions of the cross sections are identical. The CMS result is consistent with the result of the ATLAS collaboration and extends the ATLAS measurement by 0.4 unit of gap size.

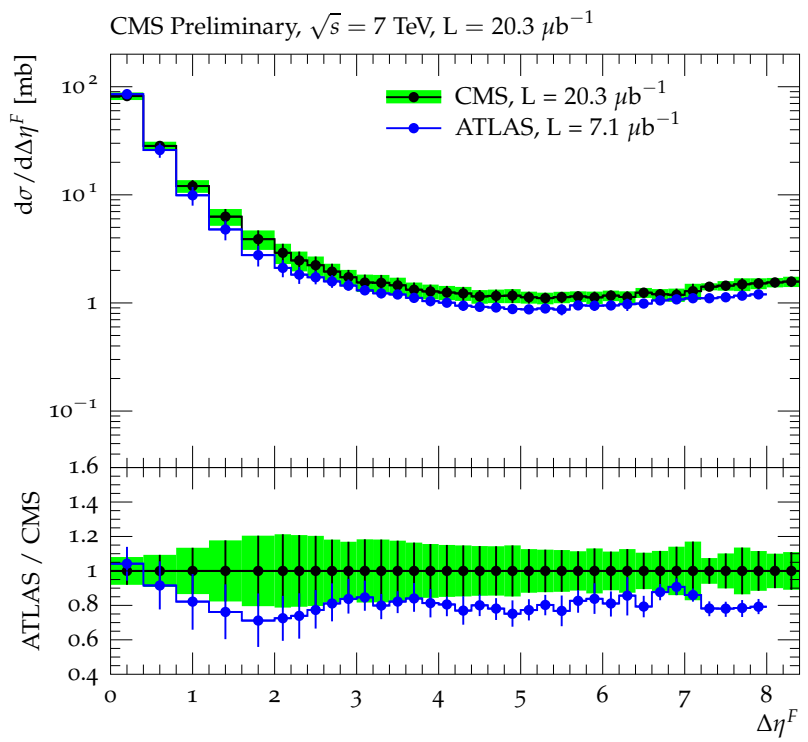

Figure 7: Comparison of the CMS and ATLAS measurements of the forward rapidity gap cross section $d \sigma / d \Delta \eta^{F}$. The band represents the total systematic uncertainty of the CMS measurement, while the total uncertainty of the ATLAS result is shown by the error bars.

\section{Diffractive dijet cross section}

The observation of a diffractive component to the dijet production cross section [31] is based on a sample of $p p$ collisions at $\sqrt{s}=7 \mathrm{TeV}$ selected online by a single jet trigger with a $p_{T}$ threshold of $6 \mathrm{GeV}$. The data sample was collected when the LHC was operating at low instantaneous luminosity and corresponds to an integrated luminosity of $2.7 \mathrm{nb}^{-1}$, with an average pileup of 0.09. This sample is dominated by nondiffractive inclusive dijet events and the selection of a diffractive-enhanced sample is based on the presence of a LRG in the event. The cross section is measured as a function of the variable $\tilde{\xi}$ that coincides with the fractional momentum loss of the scattered proton, $\xi$, for SD events.

The following requirements are applied offline. Events with at least two jets with transverse momentum $p_{T}>20 \mathrm{GeV}$ and $|\eta|<4.4$ are selected. Jets are reconstructed from particle candidates with the anti- $k_{T}$ jet clustering algorithm [48] with a distance parameter $R=0.5$. At least one primary vertex is required to be present within $24 \mathrm{~cm}$ of the nominal interaction point along the beam direction. The diffractive-enhanced sample is defined by the selections $\eta_{\max }<3$ and $\eta_{\min }>-3$, respectively. This condition corresponds to the presence of a pseudorapidity 
gap of at least 1.9 units within the acceptance of the detector, $|\eta|<4.9$. The variable $\tilde{\xi}$ is reconstructed from the energy $E^{i}$ and the longitudinal momentum $p_{z}^{i}$ of the particle candidates measured in the central detector, according to the relation $\tilde{\xi}^{ \pm}=\sum\left(E^{i} \pm p_{z}^{i}\right) / \sqrt{s}$. The variables $\tilde{\xi}^{+}$and $\tilde{\xi}^{-}$are associated to events in which the dissociated system is measured on the negative and positive $z$-side of CMS, respectively.

The distributions of the transverse momentum and pseudorapidity of the leading and secondleading jets are presented in figure 8 and compared to predictions of the MC event generators PYTHIA6 with tune Z2 [49] and PYTHIA8 with tune 1 [41]. The MC predictions are given for the nondiffractive component only. Both PYTHIA6-Z2 and PYTHIA8 tune 1 describe the transverse momentum distributions equally well, while the pseudorapidity distributions are better described by the predictions of PYTHIA6-Z2.
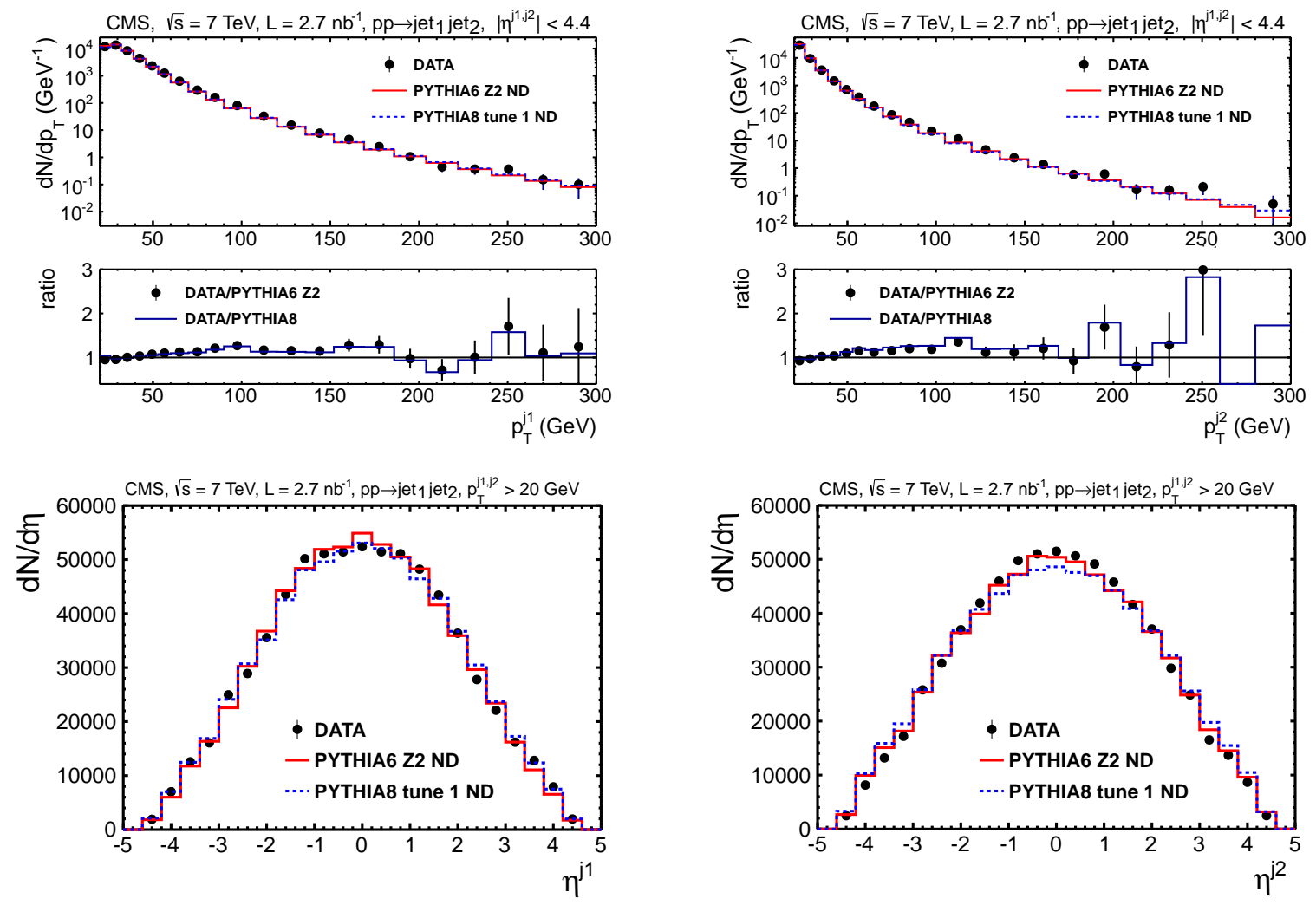

Figure 8: Detector-level distributions of the transverse momentum and pseudorapidity of the leading and second-leading jets. The data are compared to predictions of the MC event generators PYTHIA6-Z2 and PYTHIA8 tune 1. The MC predictions are given for the nondiffractive component only. The error bars indicate the statistical uncertainty. The MC predictions are normalized to the number of events in the data.

The distribution of the variable $\tilde{\xi}$ is presented in figure 9 before the $\eta_{\max }$ or $\eta_{\min }$ selection and compared to predictions of different MC event generators. The data are described by a combination of diffractive and nondiffractive contributions. The relative diffractive component and the overall normalisation factor are determined by minimising the difference between the 
distribution of the data and the sum of the nondiffractive and diffractive $\mathrm{MC}$ predictions. The data are compared in figure 9(a) to a combination of the MC event generators PYTHIA6-Z2 and POMPYT [50] that describe the nondiffractive and single diffractive components, respectively. According to the minimization procedure, the predictions of POMPYT need to be scaled by a factor 0.23 to describe the data. Figure 9(b) compares the data to a combination of PYTHIA6-D6T and POMPYT. The scale factor to be applied to the POMPYT normalisation has a value of 0.17 for this combination. The data are compared in figure $9(\mathrm{c})$ to the predictions of PYTHIA8 tune 1 . The ND, SD and DD contributions are all simulated by PYTHIA8, and the diffractive components need to be scaled by a factor 2.5 in that case. The diffractive generators POMPYT and PYTHIA 8 use the dPDFs determined by the H1 collaboration (H1 fit B) [11]. The parametrisation of the Pomeron flux in POMPYT is also based on the QCD fits to the HERA data [11], while PYTHIA8 uses a different normalisation of the Pomeron flux [51]. The large difference between the scale factors in POMPYT and PYTHIA8 is a consequence of this difference in the implementation of the Pomeron flux. None of the diffractive generators include the presence of the rapidity gap survival probability. In the case of POMPYT, the scale factor to be applied is a consequence of this fact. In the case of PYTHIA8, the normalisation of the Pomeron flux is about a factor 10 higher than in POMPYT in the low-mass region. Taking this difference into account, the scale factor to be applied to the diffractive components in PYTHIA8 would have a value of about 0.25 . The uncertainty of the scale factors is estimated by changing the fitting procedure and is found to be $\sim 20 \%$. The three different values are therefore compatible within the uncertainty.

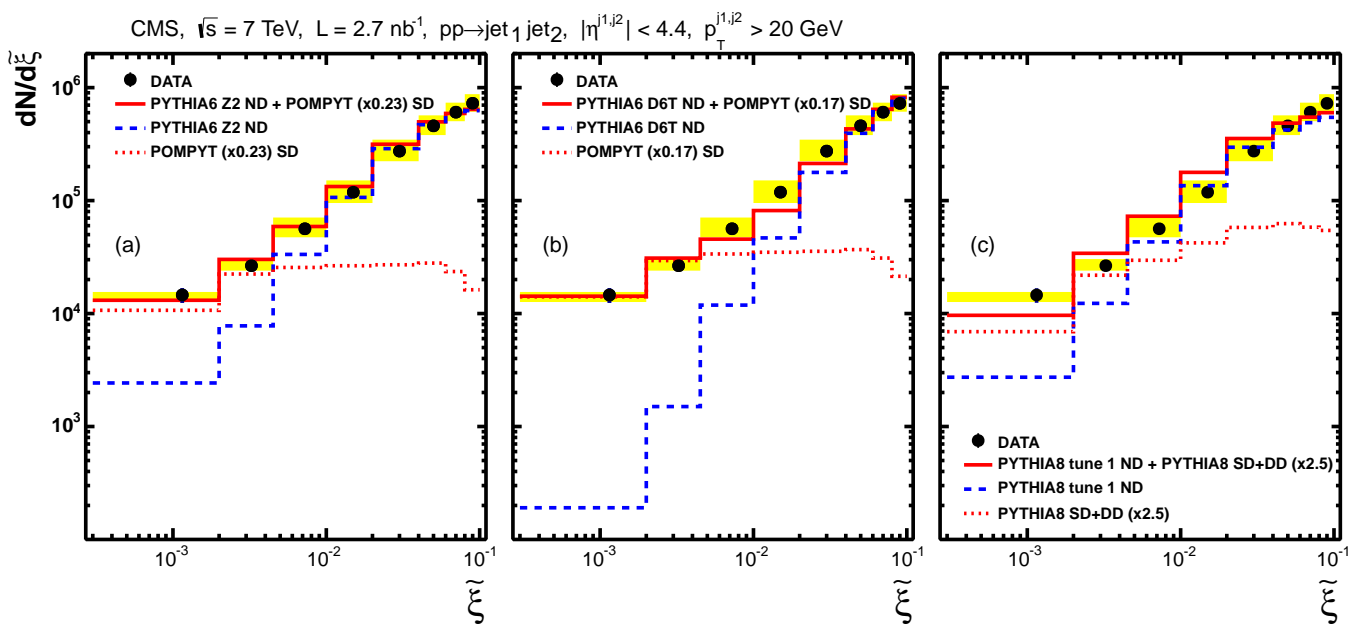

Figure 9: Detector-level $\tilde{\xi}$ distribution. The data are compared to the combinations of different MC event generators. The predictions of PYTHIA6-Z2 + POMPYT (a), PYTHIA6-D6T + POMPYT (b) and PYTHIA8 tune 1 (c) are shown. The relative diffractive contributions are scaled by the values given in the legend. The error bars indicate the statistical uncertainty, and the band represents the uncertainty on the calorimeter energy scale. The sum of the MC predictions is normalized to the number of events in the data.

The pseudorapidity distributions of the two leading jets after the selection $\eta_{\max }<3$ or $\eta_{\min }>-3$ are presented in figure 10 . The requirement of the pseudorapidity gap enhances the diffractive component in the data. The asymmetry observed in the pseudorapidity distributions 
is a consequence of the diffractive topology, the jets being mainly produced in the hemisphere opposite to that of the gap in SD events. The data are compared to a combination of the MC event generators PYTHIA6-Z2 and POMPYT, with the normalisation of the diffractive component scaled by a factor 0.23 as determined by the fitting procedure previously described.
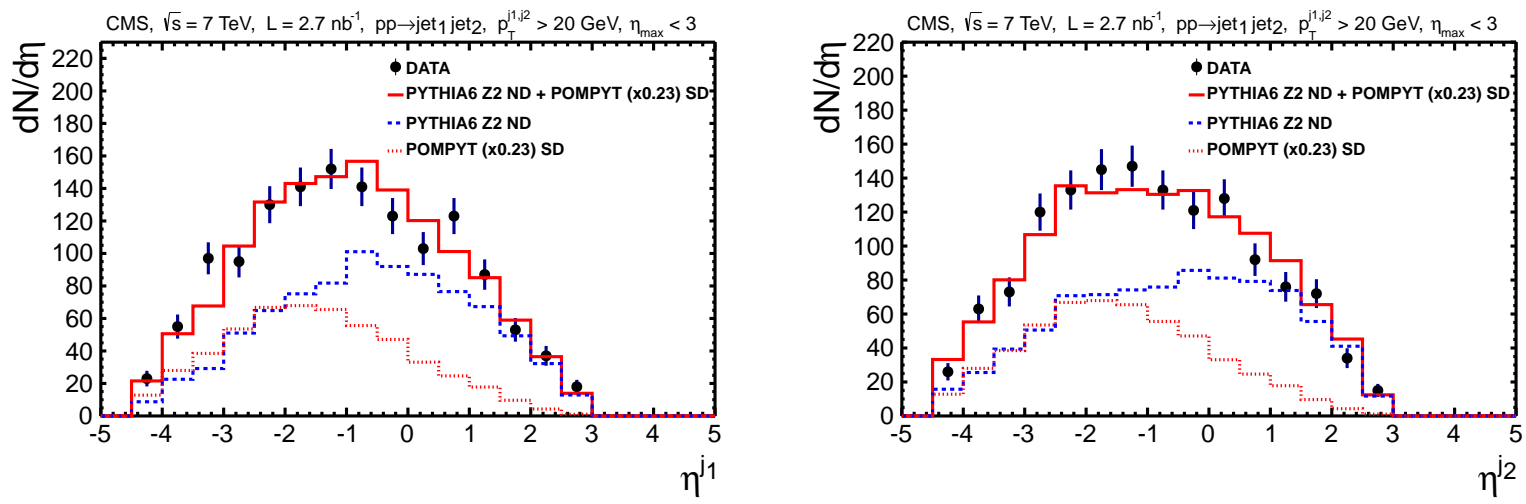

Figure 10: Detector-level pseudorapidity distributions of the leading and second-leading jets after the selection $\eta_{\max }<3$ or $\eta_{\min }>-3$. The data are compared to a combination of PYTHIA6-Z2 and POMPYT that describe the nondiffractive and single diffractive components, respectively. The individual MC predictions are also shown. The relative diffractive contribution is scaled by a factor 0.23 . The error bars indicate the statistical uncertainty. The sum of the MC predictions is normalized to the number of events in the data.

The distribution of the variable $\tilde{\xi}$ is presented in figure 11 before and after the selection $\eta_{\max }<3$ or $\eta_{\min }>-3$. The data are compared to a combination of PYTHIA6-Z2 and POMPYT, with the normalisation of POMPYT scaled by 0.23 . The requirement of the pseudorapidity gap mainly rejects events in the region of high $\tilde{\xi}$ values dominated by the ND contribution. The region of low $\tilde{\xi}$ values where the diffractive contributions dominate is only slightly affected by the selection.

The differential cross section for inclusive dijet production as a function of $\tilde{\xi}$ is corrected for the detector acceptance and the migration of reconstructed variables by a bin-by-bin unfolding procedure based on a combination of PYTHIA6-Z2 and POMPYT, with the normalisation of POMPYT scaled by 0.23 . The bin-by-bin corrections are found to be consistent with that obtained with the SVD [52] and Bayesian [53] unfolding techniques. The data are also corrected for the trigger efficiency and the effect of pileup. The total systematic uncertainty of the measurement is $\sim 30 \%$ and dominated by the uncertainty on the jet energy scale. The differential cross section is presented in figure 12 for jets with transverse momentum $p_{T}>20 \mathrm{GeV}$ and $|\eta|<4.4$. The data are compared to predictions of leading-order (LO) MC event generators, PYTHIA6Z2 and PYTHIA8 tune 1 for the ND contribution, and POMPYT, POMWIG [54] and PYTHIA8 tune 1 for the diffractive contribution. The generators POMPYT and POMWIG simulate only the SD component, while PYTHIA8 simulates both the SD and DD components. The next-to-leading-order (NLO) prediction based on POWHEG [55] is also shown in the lowest $\tilde{\xi}$ bin only. 


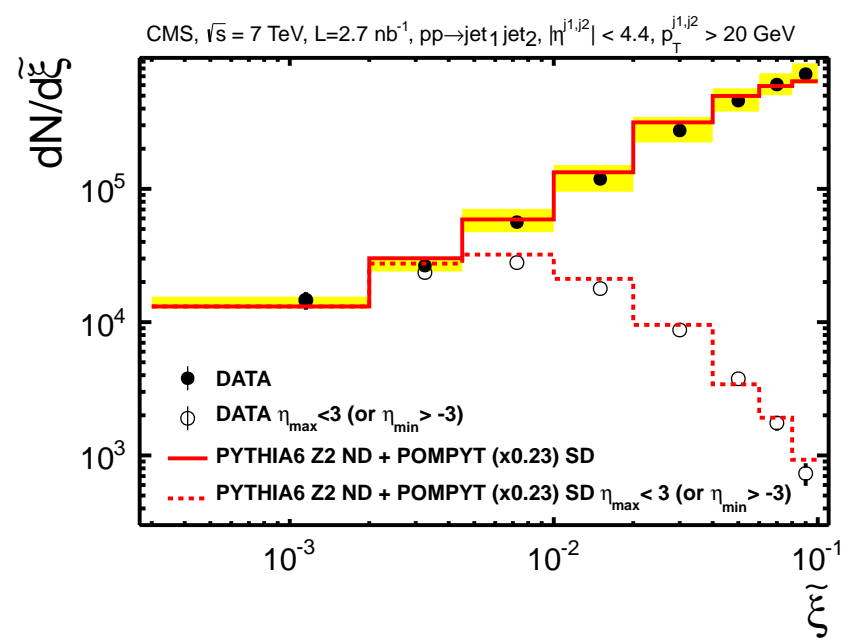

Figure 11: Detector-level $\tilde{\xi}$ distribution before and after the rapidity gap selection $\eta_{\max }<3$ or $\eta_{\min }>-3$. The data are compared to a combination of PYTHIA6-Z2 and POMPYT. The relative diffractive contribution is scaled by a factor 0.23 . The error bars indicate the statistical uncertainty, and the band represents the calorimeter energy scale uncertainty. The sum of the MC predictions is normalized to the number of events in the data.

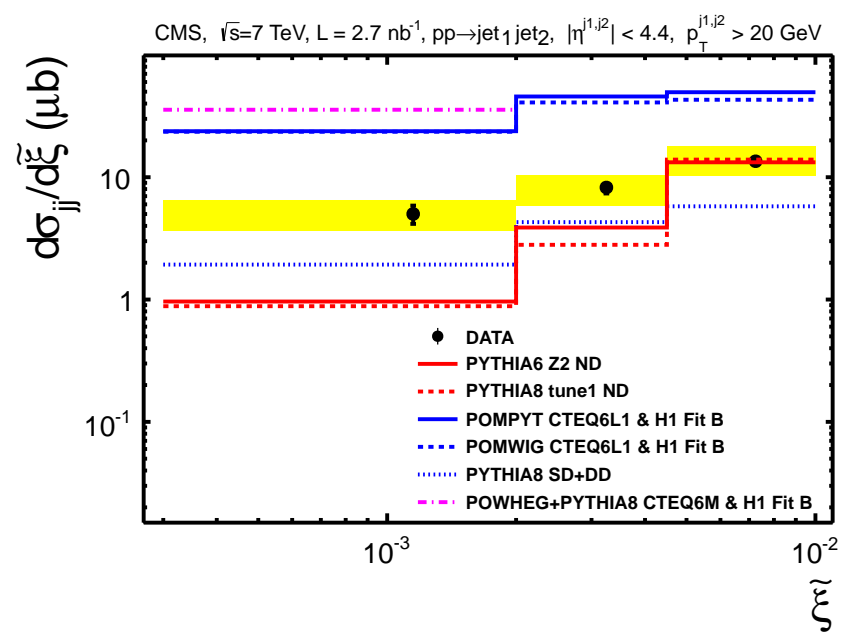

Figure 12: Differential cross section for inclusive dijet production as a function of $\tilde{\xi}$ for jets with transverse momentum $p_{T}>20 \mathrm{GeV}$ and $|\eta|<4.4$. The error bars indicate the statistical uncertainty and the band represents the systematic uncertainties added in quadrature. The data are compared to predictions of LO MC event generators, PYTHIA6-Z2 and PYTHIA8 tune 1 for the ND contribution, and POMPYT, POMWIG and PYTHIA8 tune 1 for the diffractive contribution. The generators POMPYT and POMWIG simulate only the SD component, while PYTHIA8 simulates both the SD and DD components. The NLO prediction based on POWHEG is also shown in the lowest $\tilde{\xi}$ bin only. 
The generators PYTHIA8, POMPYT and POMWIG, and the NLO predictions based on the POWHEG framework use the dPDFs determined by the H1 collaboration (H1 fit $\mathrm{B}$ [11]). The main difference between POMPYT and POMWIG is that POMPYT uses the PYTHIA framework for the hadronization, while POMWIG is based on the HERWIG [56] framework. The predictions of PYTHIA6-Z2 and PYTHIA8 tune 1 for the ND contribution underestimate the data in the low $\tilde{\xi}$ region. The predictions of POMPYT, POMWIG and POWHEG are needed to describe the low $\tilde{\xi}$ region but overestimate the data by a factor $\sim 5$. The ratio of the measured cross section to that expected from the diffractive MC models gives an upper limit of the rapidity gap survival probability, the measured cross section including a proton dissociation contribution that needs to be subtracted to get an estimate of the survival probability. For POMPYT and POMWIG, the value of the upper limit is $0.21 \pm 0.07$. After correction for the proton dissociation contribution present in the measured cross section and in the dPDFs, an estimate of the rapidity gap survival probability can be obtained and is found to be $0.12 \pm 0.05$ for the LO MC generators POMPYT and POMWIG. If the NLO predictions based on POWHEG are used, the estimation of the rapidity gap survival probability gives a lower value of $0.08 \pm 0.04$. It should be mentioned that the $\mathrm{dPDF}$ and the proton dissociation contribution are poorly constrained in the region of the measurement. This contribution to the uncertainty of the survival probability is not taken into account. The normalisation of the diffractive predictions of PYTHIA8 disagrees with that of POMPYT and POMWIG, and the predictions would need to be scaled by a factor $\sim 2.5$ to describe the data. This is a consequence of the different normalisation of the Pomeron flux in PYTHIA8 with respect to that used in the $\mathrm{H} 1$ fit B. For that reason, PYTHIA8 can not directly be used to estimate the gap survival probability. The present value is compatible with the CDF measurement $[18,20]$.

\section{$8 W$ and $Z$ boson events with a LRG signature}

The observation of $W$ and $Z$ boson events with a large pseudorapidity gap [32] is based on a sample of $p p$ collisions at $\sqrt{s}=7 \mathrm{TeV}$ selected online by requiring a high transverse momentum electron or muon with $p_{T}$ thresholds varying between 10 and $17 \mathrm{GeV}$ for electrons and between 9 and $15 \mathrm{GeV}$ for muons. The data sample was collected when the LHC was steadily increasing the instantaneous luminosity and corresponds to an integrated luminosity of $36 \mathrm{pb}^{-1}$. The average pileup is increasing from less than one additional inelastic $p p$ collision per bunch crossing to more than two. This sample is dominated by inclusive $W$ and $Z$ boson events and the selection of a diffractive-enhanced sample is based on the presence of a LRG in the event.

The identification of $W$ and $Z$ bosons is based on the presence of isolated electrons and muons with high transverse momentum. The following requirements are applied offline. In order to limit the effects of pileup, events with more than one vertex are rejected. Isolated electron and muon candidates are selected with a transverse momentum $p_{T}>25 \mathrm{GeV}$ and $|\eta|<2.5$. Electrons from photon conversions are rejected. Muons are required to be identified by two different reconstruction algorithms [57]. Jets and missing transverse momenta are determined from particle candidates. For the jet reconstruction, the anti- $k_{T}$ jet clustering algorithm [48] is used with a distance parameter $R=0.5$. Jets are selected with a transverse momentum $p_{T}>30 \mathrm{GeV}$ and $|\eta|<2.5$. 
An event is selected as a $W \rightarrow l \nu$ candidate if it fulfills the following requirements. One isolated electron or muon with $p_{T}>25 \mathrm{GeV}$ and $|\eta|<1.4$ is present in the event, and no other isolated electron or muon with $p_{T}>10 \mathrm{GeV}$ is found. The missing transverse momentum is greater than $30 \mathrm{GeV}$ and the transverse mass of the charged lepton and the neutrino greater than $60 \mathrm{GeV}$. An event is selected as a $Z \rightarrow l l$ candidate if the following requirements are satisfied. Two isolated electrons or muons with opposite charge and $p_{T}>25 \mathrm{GeV}$ are present in the event. At least one of the leptons has $|\eta|<1.4$ and the invariant mass of the dilepton system is between 60 and $120 \mathrm{GeV}$.

The presence of a LRG is defined by the absence of activity above the noise threshold in at least one of the HF calorimeters. This condition defines the diffractive-enhanced samples of $W$ and $Z$ boson events and corresponds to the presence of a pseudorapidity gap of at least 1.9 units in the event, the fiducial coverage of the HF calorimeters being limited to the region $3<|\eta|<4$.9. Soft pileup events with few or no activity in the central region of the detector are not rejected by the single vertex requirement and have the ability to fill the pseudorapidity gap in the forward region. The contribution of these events to the energy measured in the HF calorimeters is found to be well reproduced by the CMS simulation.

Diffractively produced $W$ and $Z$ bosons are more likely to be found in the hemisphere opposite to that of the gap. This is a consequence of the fact that dPDFs peak at smaller $x$ values than the inclusive parton distribution functions (PDFs). The boson produced being boosted in the direction of the parton with the highest momentum fraction, it is more likely to be found in the direction of the dissociated system, opposite to that of the gap. This property is used to estimate the diffractive component in the samples of $W$ and $Z$ boson events with a LRG.

The distribution of the signed pseudorapidity of the charged lepton, $\eta_{l}$, in $W$ events with a LRG is shown in figure 13 for the combination of the electron and muon channels. The sign is defined according to the hemispheres in which the pseudorapidity gap and the charged lepton are found. The pseudorapidity is positive when the gap and the lepton are in the same hemisphere and negative otherwise. A total of 147 events are found with the charged lepton in the hemisphere opposite to that of the gap, and 96 events with the lepton in the same hemisphere. The asymmetry can be defined as the ratio of the difference between the numbers of LRG events with positive and negative $\eta_{l}$ and the total number of events. The corresponding asymmetry is $21.0 \pm 6.4 \%$. For the sample of $Z$ bosons with a LRG, a compatible asymmetry of $20 \pm 16 \%$ is found. The data are compared to a combination of the MC event generators PYTHIA6 Pro-Q20 [58] and POMPYT that describe the nondiffractive and single diffractive components, respectively. The predictions of POMPYT show a strong asymmetry in $\eta_{l}$, while the nondiffractive contribution estimated with PYTHIA6 Pro-Q20 is symmetric. The relative diffractive contribution is determined by minimising the difference between the distribution of the data and the sum of the nondiffractive and diffractive MC predictions. According to the minimization procedure, the fraction of diffractive events in the sample of $W$ events with a LRG is found to be $(50.0 \pm 9.3$ (stat.) \pm 5.2 (syst.) $) \%$. The choice of PYTHIA6 Pro-Q20 to describe the nondiffractive component comes from the fact that this generator gives the best description of the energy distribution in the HF calorimeters, especially at low energy. The minimization procedures based on the combination of POMPYT with PYTHIA6-D6T, PYTHIA6-Z2 or 
PYTHIA8 2C [42] give similar results. The nondiffractive predictions of these generators are shown in figure 13. The systematic uncertainty of $5.2 \%$ is estimated from the uncertainty on the HF calorimeter energy scale and the choice of different nondiffractive predictions in the fits.

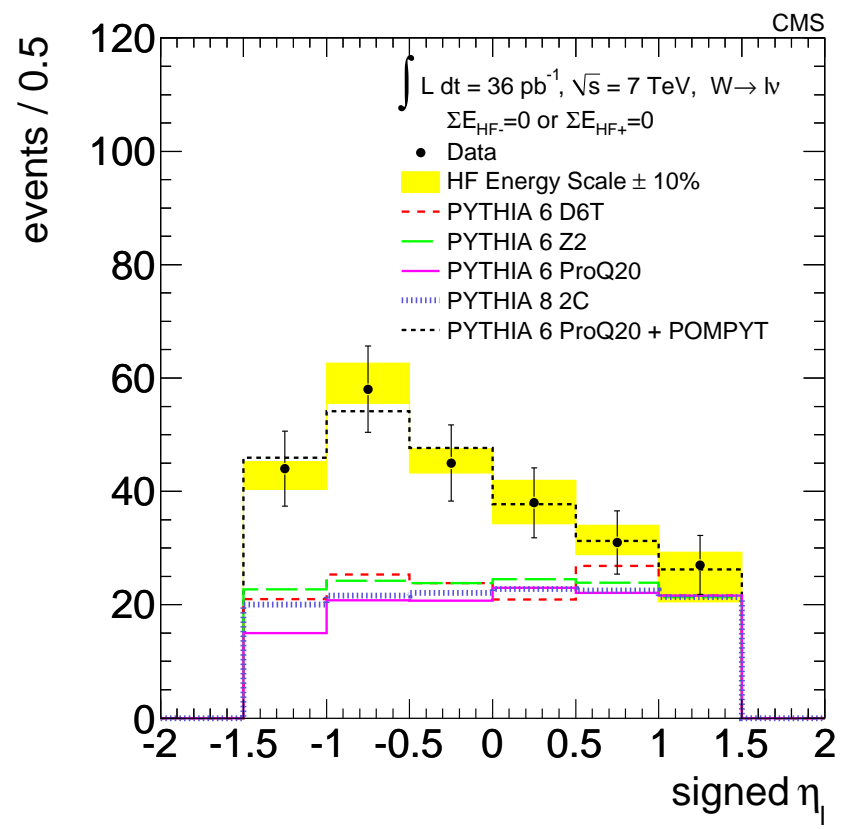

Figure 13: Distribution of the signed pseudorapidity of the charged lepton, $\eta_{l}$, in $W$ events with a LRG for the combination of the electron and muon channels. The pseudorapidity is positive when the gap and the lepton are in the same hemisphere and negative otherwise. The data are compared to a combination of PYTHIA6 Pro-Q20 and POMPYT that describe the nondiffractive and single diffractive components, respectively. The nondiffractive predictions are also shown for the MC generators PYTHIA8 2C and PYTHIA6 with tunes D6T, Z2 and Pro-Q20. The band represents the energy scale uncertainty of the HF calorimeters.

\section{Pseudorapidity distribution of charged particles in a single diffractive enhanced sample}

The measurement of pseudorapidity distributions of charged particles [33] is based on a sample of $p p$ collisions at $\sqrt{s}=8 \mathrm{TeV}$ collected in July 2012 by a minimum-bias trigger during a dedicated run, when the LHC was operating in a very low pileup scenario and with a non-standard $\beta^{*}=90 \mathrm{~m}$ optics configuration, with $\beta^{*}$ the amplitude function of the beam at the IP. These data correspond to an integrated luminosity of $45 \mu \mathrm{b}^{-1}$ with an average number of inelastic $p p$ collisions per bunch crossing of 0.04. The minimum-bias trigger is provided by the TOTEM T2 telescopes. It contributes to the CMS global trigger decision and initiates the simultaneous readout of the CMS and TOTEM detectors. The trigger requires the presence of at least one track candidate in one of the T2 telescopes in either side of the IP. Events reconstructed by the CMS and TOTEM detectors are combined offline. 
The pseudorapidity distributions of charged particles $\mathrm{d} N_{\mathrm{ch}} / \mathrm{d} \eta$ are measured in the forward region covered by the TOTEM T2 telescopes $(5.3<|\eta|<6.4)$ for primary tracks with $p_{T}>40 \mathrm{MeV}$, and in the central region covered by the CMS tracker $(|\eta|<2.2)$ for primary tracks with $p_{T}>100 \mathrm{MeV}$. In order to limit the effects of pileup, events with more than one reconstructed vertex are rejected. The pseudorapidity distributions are measured for 3 different event topologies selected offline by the following requirements. A sample of inclusive inelastic events is defined by the presence of at least one primary track candidate in one of the T2 telescopes in either side of the IP. A sample enhanced in non single diffractive dissociation (NSD) events is selected by requiring at least one primary track candidate in both T2 telescopes, and a sample enriched in single diffractive dissociation (SD) events is obtained by requiring at least one primary track candidate in one T2 telescope and none in the other. For the completeness of the discussion, the distributions associated to the 3 different topologies are presented.

The $\mathrm{d} N_{\mathrm{ch}} / \mathrm{d} \eta$ distributions are corrected for the primary track reconstruction, the vertex reconstruction and selection, the event selection, the misidentification of secondary tracks as primary ones and the multiple reconstruction of single charged particles. The final measurements are extrapolated down to $p_{T}=0$ with correction factors determined in a MC-driven way from the $p_{T}$ spectrum of primary charged particles. For the measurement in the central region, the dominant sources of systematic uncertainties for the inclusive and NSD-enhanced samples are the track reconstruction and the event selection. The total systematic uncertainty ranges from 5 to $7 \%$ and 6 to $8 \%$ for the inclusive and NSD-enhanced samples, respectively. For the SD-enhanced sample, the dominant systematic uncertainties are due to the uncertainty in the event selection and the model dependence of the event selection efficiency. The total systematic uncertainty is between 10 and $17 \%$. For the measurement in the forward region, the dominant sources of systematic uncertainties for the inclusive and NSD-enhanced samples are the primary track selection, the track reconstruction and the description of the material between the T2 telescopes and the IP. The total systematic uncertainty ranges from 10 to $12 \%$ for both samples. For the SD-enhanced sample, the most significant systematic uncertainty is due to the event selection, and the total systematic uncertainty is between 16 and $18 \%$.

The combined CMS-TOTEM measurement of pseudorapidity distributions of charged particles is presented in figure 14. The results are obtained by averaging the data points in the corresponding positive and negative $\eta$ bins. The band represents the total uncertainty, while the error bars indicate the statistical and uncorrelated systematic uncertainties added in quadrature. The data are compared to predictions of the MC event generators PYTHIA6-Z2*, PYTHIA8-4C and HERWIG ++ [59] that has a recent tune to LHC data, UE-EE-3 [60]. The data are also compared to predictions of two $\mathrm{MC}$ event generators used in cosmic ray physics, EPOS [61] with the LHC tune [62] and QGSJetII-04 [63]. These two MC models include contributions from soft and hard parton dynamics. The soft component is described in terms of the exchange of virtual quasi-particle states, while the hard component is included via hard-Pomeron scattering diagrams, which are equivalent to a leading-order perturbative QCD approach with DGLAP evolution. The models used in EPOS and QGSJETII-04 are retuned to LHC data [64]. None of the models considered provide a consistent description of the measured distributions. In the central region, the predictions from the various $\mathrm{MC}$ event generators differ from the data by up to $20 \%$ for the inclusive and NSD-enhanced samples, with even 

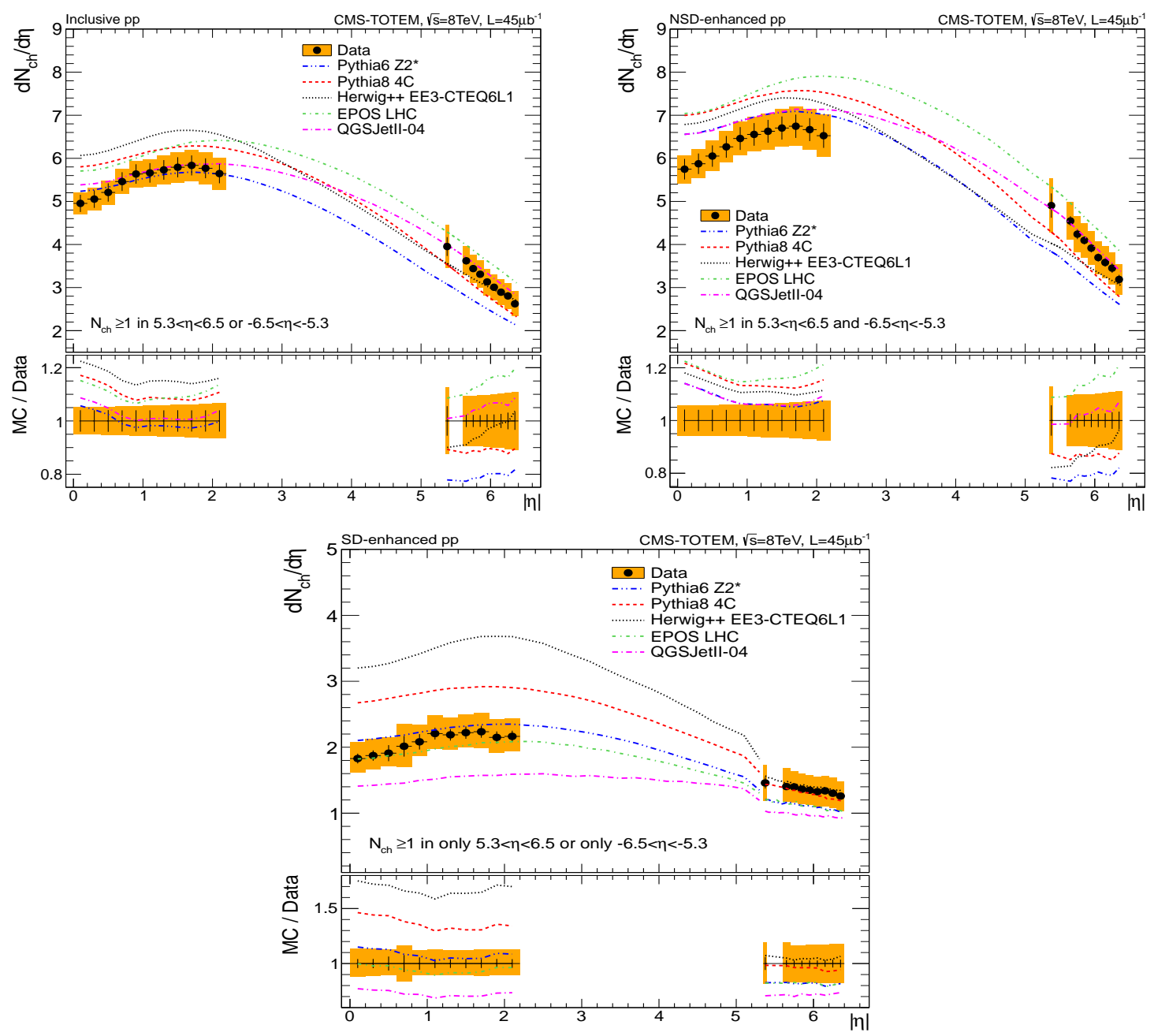

Figure 14: Pseudorapidity distributions of charged particles for an inclusive sample (top left), a NSD-enhanced sample (top right), and a SD-enhanced sample (bottom). The error bars indicate the statistical and uncorrelated systematic uncertainties added in quadrature, while the bands show the total uncertainty. The data are compared to predictions of the MC event generators PYTHIA6-Z2*, PYTHIA8-4C, HERWIG++ with tune UE-EE-3, EPOS with tune LHC, and QGSJETII-04.

larger discrepancies for the SD-enhanced sample. For the inclusive sample, the data are well described by PYTHIA6 and QGSJETII-04 . For the NSD-enhanced sample, the predictions from PYTHIA6 and QGSJETII-04 agree with the data for most $\eta$ bins. For the SD-enhanced sample, both EPOS and PYTHIA6 provide a good description of the measurement. In the forward region, the MC predictions differ from the data by up to $30 \%$ for the three samples. For the inclusive and NSD-enhanced samples, the data are in agreement with the predictions from QGSJETII-04, and between the EPOS and PYTHIA8 results. For the SD-enhanced sample, the TOTEM data points are close to the PYTHIA 8 and HERWIG ++ predictions, while QGSJETII-04 underestimates the data. 


\section{Prospects of common CMS-TOTEM data taking}

During the LHC Run 1, diffractive processes have been mainly selected by requiring a large rapidity gap in the event. As previously mentioned, the successful collaboration between the CMS and TOTEM experiments has recently enabled us to complement the central CMS measurement with the scattered proton information, measured with the TOTEM Roman pots, in dedicated common runs during 2012 and 2013. Tagging of the scattered proton(s) enables to measure the four-momentum squared exchanged at the proton vertex, $t$, as well as the fractional momentum loss of the proton, $\xi$, when studying SD and CEP processes, and to reduce significantly the proton dissociation background that is present in measurements with the CMS central detector only. In the case of CEP, tagging the scattered protons allows reconstructing the mass of the centrally produced system with a resolution not achievable with the central detector only.

A common CMS-TOTEM project for a precision proton spectrometer (CT-PPS) [65] has now been approved, with installation planned at the end of the ongoing Long Shutdown 1 (LS1). This project consists of tracking and timing detectors and is comprised of two phases, with the first taking place in 2015 at the start of the LHC Run 2, and the second expected to start one year later. Data will be collected at high instantaneous luminosity with a standard low $\beta^{*}$ value of $0.5 \mathrm{~m}$. The collected luminosity is expected to be of the order of $100 \mathrm{fb}^{-1}$ before the start of the Long Shutdown 2 (LS2) in 2018.

In Phase I of the CT-PPS project, data will be collected by making use of the existing $147 \mathrm{~m}$ TOTEM horizontal Roman pots, relocated in the $200-225 \mathrm{~m}$ region and instrumented with silicon strip tracking detectors. The tracking system will be complemented by timing detectors located in a new cylindrical Roman pot, which make it possible to identify the vertex associated to the scattered protons. At high instantaneous luminosity, each crossing of the proton beams has an increased probability to produce several $p p$ collisions, and the availability of the timing detectors is critical in the presence of a high pileup environment. The data acquisition of TOTEM is expected to be fully integrated with that of CMS, opening the possibility to combine CMS and TOTEM information at trigger level. This first phase will be essentially dedicated to the understanding of the detector and backgrounds. The silicon strip detectors being not able to survive a continuous data taking at high instantaneous luminosity, they will be mainly used to perform rate measurements during short insertions at the end of the fills. The integrated luminosity collected during the first phase of the CT-PPS project will only correspond to a small fraction of the expected $100 \mathrm{fb}^{-1}$.

In Phase II of the CT-PPS project, the silicon strip tracking detectors will be replaced by radiation hard pixel detectors. This second phase will be devoted to the study of small cross section high mass CEP processes with the mass of the centrally produced system higher than $300 \mathrm{GeV}$. Central exclusive production provides a unique method to access a variety of physics topics, such as new physics via anomalous production of $W$ and $Z$ boson pairs, high transverse momentum dijet production, and possibly the production of new resonances.

Apart from the CT-PPS project, for which the hardware is jointly built, managed and 
operated by CMS and TOTEM together, the TOTEM collaboration will pursue in 2015 a high cross section forward physics programme at low instantaneous luminosity [66]. The collected luminosity is expected to be of the order of $1-10 \mathrm{pb}^{-1}$ for a high $\beta^{*}$ value of $90 \mathrm{~m}$. These special runs will be supported by CMS as common runs in terms of trigger and detector readout, in order to complement the central CMS measurement with the scattered proton information measured with the TOTEM Roman pots. The physics programme is dedicated to the study of low mass SD and CEP processes, with the mass of the diffractive or centrally produced system smaller than $100 \mathrm{GeV}$. These processes include the single diffractive production of a dijet system, $J / \Psi$ or $W$ boson, the CEP production of a dijet system, and the search for glueballs.

\section{Summary}

A review of the CMS results on diffraction has been presented. These include the measurements of the soft diffractive cross sections [30], of the forward rapidity gap cross section [30], of the diffractive dijet cross section [31], the measurement of a large rapidity gap in $W$ and $Z$ boson events [32], and the measurement of the pseudorapidity distribution of charged particles in a single diffractive enhanced sample [33]. This last measurement is the first common result of the CMS and TOTEM collaborations. Given the importance of the diffractive contribution to the UE and the need to constrain its phenomenological description, the measurement of the soft diffractive cross sections is of primary importance. The use of the forward CASTOR calorimeter has enabled us to measure both the SD and DD cross sections, which provide valuable input to constrain the modelling of hadronization and diffraction. The measurement of the forward rapidity gap cross section is consistent with the result of the ATLAS collaboration [47] and extends the ATLAS measurement by 0.4 unit of gap size. The first observation of a diffractive contribution to dijet production at the LHC has enabled to obtain an estimate of the rapidity gap survival probability compatible with the CDF measurement $[18,20]$. The use of the LO MC event generators POMPYT and POMWIG gives a value of $0.12 \pm 0.05$, while the use of the NLO predictions based on POWHEG gives a lower value of $0.08 \pm 0.04$. The measurement of a large rapidity gap in $W$ and $Z$ boson events is the first observation of a diffractive contribution to $W$ and $Z$ bosons production at the LHC. The relative diffractive contribution in a sample of $\mathrm{W}$ boson events with a $\mathrm{LRG}$ is found to be $(50.0 \pm 9.3$ (stat.) \pm 5.2 (syst.))\% when using a combination of the MC event generators PYTHIA6 Pro-Q20 and POMPYT to describe the nondiffractive and single diffractive components, respectively. The measurement of pseudorapidity distributions of charged particles covers the largest pseudorapidity interval ever measured at the LHC and has the potential to probe the correlation between particle production in the central and forward regions. None of the models considered provide a consistent description of the measurement over the whole $\eta$ range and for the 3 different event samples.

\section{Acknowledgements}

The author would like to thank the members of the diffractive and exclusive group of the CMS collaboration, as well as the members of the TOTEM collaboration involved in the common 
CMS-TOTEM analyses. A special thank to Pierre Van Mechelen, Hans Van Haevermaet, Robert Ciesielski and Kenneth Österberg for the fruitful discussions on the ongoing analyses.

\section{References}

[1] N. Cartiglia, "Measurement of the proton-proton total cross section at 2, 7, 8 and $57 \mathrm{TeV}$ " [arXiv:1303.2927 [hep-ph]].

[2] V. Barone, "High-energy particle diffraction", Berlin, Springer 2002, 407 p.

[3] M. Arneodo and M. Diehl, "Diffraction for non-believers" [arXiv:0511047 [hep-ph]].

[4] P. D. B. Collins, "An Introduction to Regge Theory and High-Energy Physics", Cambridge 1977, $445 \mathrm{p}$.

[5] J. C. Collins, "Proof of factorization for diffractive hard scattering", Phys. Rev. D 57 (1998) 3051 [Erratum-ibid. D 61 (2000) 019902] [arXiv:9709499 [hep-ph]].

[6] A. Berera and D. E. Soper, "Behavior of diffractive parton distribution functions", Phys. Rev. D 53 (1996) 6162 [arXiv:9509239 [hep-ph]].

[7] V. N. Gribov and L. N. Lipatov, "Deep inelastic e p scattering in perturbation theory", Sov. J. Nucl. Phys. 15 (1972) 438 [Yad. Fiz. 15 (1972) 781].

[8] L. N. Lipatov, "The parton model and perturbation theory", Sov. J. Nucl. Phys. 20 (1975) 94 [Yad. Fiz. 20 (1974) 181].

[9] G. Altarelli and G. Parisi, "Asymptotic Freedom in Parton Language", Nucl. Phys. B 126 (1977) 298.

[10] Y. L. Dokshitzer, "Calculation of the Structure Functions for Deep Inelastic Scattering and e+ e- Annihilation by Perturbation Theory in Quantum Chromodynamics", Sov. Phys. JETP 46 (1977) 641 [Zh. Eksp. Teor. Fiz. 73 (1977) 1216].

[11] A. Aktas et al. [H1 Collaboration], "Measurement and QCD analysis of the diffractive deep inelastic scattering cross section at HERA", Eur. Phys. J. C 48 (2006) 715 [arXiv:0606004 [hep-ex]].

[12] S. Chekanov et al. [ZEUS Collaboration], "A QCD analysis of ZEUS diffractive data", Nucl. Phys. B 831 (2010) 1 [arXiv:0911.4119 [hep-ex]].

[13] S. Chekanov et al. [ZEUS Collaboration], "Deep inelastic scattering with leading protons or large rapidity gaps at HERA", Nucl. Phys. B 816 (2009) 1 [arXiv:0812.2003 [hep-ex]].

[14] F. D. Aaron et al. [H1 and ZEUS Collaborations], "Combined inclusive diffractive cross sections measured with forward proton spectrometers in deep inelastic ep scattering at HERA", Eur. Phys. J. C 72 (2012) 2175 [arXiv:1207.4864 [hep-ex]]. 
[15] F. D. Aaron et al. [H1 Collaboration], "Inclusive Measurement of Diffractive Deep-Inelastic Scattering at HERA”, Eur. Phys. J. C 72 (2012) 2074 [arXiv:1203.4495 [hep-ex]].

[16] F. D. Aaron et al. [H1 Collaboration], "Diffractive Dijet Photoproduction in ep Collisions at HERA", Eur. Phys. J. C 70 (2010) 15 [arXiv:1006.0946 [hep-ex]].

[17] F. D. Aaron et al. [H1 Collaboration], "Measurement of Dijet Production in Diffractive Deep-Inelastic Scattering with a Leading Proton at HERA", Eur. Phys. J. C 72 (2012) 1970 [arXiv:1111.0584 [hep-ex]].

[18] T. Affolder et al. [CDF Collaboration], "Diffractive dijets with a leading antiproton in $\bar{p} p$ collisions at $\sqrt{s}=1800 \mathrm{GeV}$ ", Phys. Rev. Lett. 84 (2000) 5043.

[19] B. Abbott et al. [D0 Collaboration], "Hard single diffraction in $\bar{p} p$ collisions at $\sqrt{s}=630$ GeV and 1800 GeV", Phys. Lett. B 531 (2002) 52 [arXiv:9912061 [hep-ex]].

[20] T. Aaltonen et al. [CDF Collaboration], "Diffractive Dijet Production in $\bar{p} p$ Collisions at $\sqrt{s}=1.96$ TeV", Phys. Rev. D 86 (2012) 032009 [arXiv:1206.3955 [hep-ex]].

[21] T. Aaltonen et al. [CDF Collaboration], "Diffractive W and Z Production at the Fermilab Tevatron", Phys. Rev. D 82 (2010) 112004 [arXiv:1007.5048 [hep-ex]].

[22] A. B. Kaidalov, V. A. Khoze, A. D. Martin and M. G. Ryskin, "Factorization breaking in diffractive dijet photoproduction at HERA", Eur. Phys. J. C 66 (2010) 373 [arXiv:0911.3716 [hep-ph]].

[23] M. Klasen, "Factorization breaking in single-diffractive dijet production at the Tevatron", Nucl. Phys. Proc. Suppl. 207-208 (2010) 25 [arXiv:1007.1121 [hep-ph]].

[24] E. Gotsman, E. Levin, U. Maor, E. Naftali and A. Prygarin, "Survival probability of large rapidity gaps" [arXiv:0511060 [hep-ph]].

[25] V. A. Khoze, A. D. Martin and M. G. Ryskin, "The Extraction of the bare triple-Pomeron vertex: A Crucial ingredient for diffraction", Phys. Lett. B 643 (2006) 93 [arXiv:0609312 [hep-ph]].

[26] E. Gotsman, "Survival Probability at the LHC", AIP Conf. Proc. 1105 (2009) 213 [arXiv:0811.0491 [hep-ph]].

[27] E. Gotsman, E. Levin and U. Maor, "Survival probability of large rapidity gaps in QCD and N=4 SYM motivated model", Eur. Phys. J. C 71 (2011) 1685 [arXiv:1101.5816 [hep$\mathrm{ph}]$.

[28] P. Bartalini and L. Fano, "Multiple partonic interactions at the LHC", Proceedings, 1st International Workshop, MPI'08, Perugia, Italy, October 27-31, 2008 [arXiv:1003.4220 [hep-ex]].

[29] S. Alekhin, G. Altarelli, N. Amapane, J. Andersen, V. Andreev, M. Arneodo, V. Avati and J. Baines et al., "HERA and the LHC: A Workshop on the implications of HERA for LHC physics. Proceedings, Part B" [arXiv:0601013 [hep-ph]]. 
[30] CMS Collaboration [CMS Collaboration], "Measurement of diffraction dissociation cross sections at $\sqrt{s}=7 \mathrm{TeV}$ at the LHC", CMS-PAS-FSQ-12-005.

[31] S. Chatrchyan et al. [CMS Collaboration], "Observation of a diffractive contribution to dijet production in proton-proton collisions at $\sqrt{s}=7$ TeV", Phys. Rev. D 87 (2013) 012006 [arXiv:1209.1805 [hep-ex]].

[32] S. Chatrchyan et al. [CMS Collaboration], "Forward Energy Flow, Central ChargedParticle Multiplicities, and Pseudorapidity Gaps in W and Z Boson Events from pp Collisions at $\sqrt{s}=7$ TeV", Eur. Phys. J. C 72 (2012) 1839 [arXiv:1110.0181 [hep-ex]].

[33] S. Chatrchyan et al. [CMS and TOTEM Collaborations], "Measurement of pseudorapidity distributions of charged particles in proton-proton collisions at $\sqrt{s}=8 \mathrm{TeV}$ by the CMS and TOTEM experiments", Eur. Phys. J. C 74 (2014) 10, 3053 [arXiv:1405.0722 [hep-ex]].

[34] S. Chatrchyan et al. [CMS Collaboration], "The CMS experiment at the CERN LHC," JINST 3 (2008) S08004.

[35] V. Andreev, X. Aslanoglou, A. Azman, M. N. Bakirci, S. Basegmez, W. Beaumont, J. Blocki and K. Borras et al., "Performance studies of a full-length prototype for the CASTOR forward calorimeter at the CMS experiment", Eur. Phys. J. C 67 (2010) 601.

[36] G. Anelli et al. [TOTEM Collaboration], "The TOTEM experiment at the CERN Large Hadron Collider", JINST 3 (2008) S08007.

[37] G. Antchev et al. [TOTEM Collaboration], "Performance of the TOTEM Detectors at the LHC", Int. J. Mod. Phys. A 28 (2013) 1330046 [arXiv:1310.2908 [physics.ins-det]].

[38] M. G. Bagliesi, M. Berretti, E. Brucken, R. Cecchi, E. David, F. Garcia, V. Greco and J. Heino et al., "The TOTEM T2 telescope based on triple-GEM chambers", Nucl. Instrum. Meth. A 617 (2010) 134.

[39] G. Antchev et al. [TOTEM Collaboration], "Measurement of the forward charged particle pseudorapidity density in $p p$ collisions at $\sqrt{s}=7 \mathrm{TeV}$ with the TOTEM experiment", Europhys. Lett. 98 (2012) 31002 [arXiv:1205.4105 [hep-ex]].

[40] R. Ciesielski and K. Goulianos, "MBR Monte Carlo Simulation in PYTHIA8", PoS ICHEP 2012 (2013) 301 [arXiv:1205.1446 [hep-ph]].

[41] T. Sjostrand, S. Mrenna and P. Z. Skands, "A Brief Introduction to PYTHIA 8.1", Comput. Phys. Commun. 178 (2008) 852 [arXiv:0710.3820 [hep-ph]].

[42] R. Corke and T. Sjostrand, "Interleaved Parton Showers and Tuning Prospects", JHEP 1103 (2011) 032 [arXiv:1011.1759 [hep-ph]].

[43] T. Sjostrand, S. Mrenna and P. Z. Skands, "PYTHIA 6.4 Physics and Manual", JHEP 0605 (2006) 026 [arXiv:0603175 [hep-ph]].

[44] R. Field, "Physics at the Tevatron", Acta Phys. Polon. B 39 (2008) 2611. 
[45] T. Adye, "Unfolding algorithms and tests using RooUnfold", Proceedings of the PHYSTAT 2011 Workshop, CERN, Geneva, Switzerland, January 2011, CERN-2011-006, pp 313-318 [arXiv:1105.1160 [physics.data-an]].

[46] S. Chatrchyan et al. [CMS Collaboration], "Study of the underlying event at forward rapidity in pp collisions at $\sqrt{s}=0.9,2.76$, and $7 \mathrm{TeV}$ ", JHEP 1304 (2013) 072 [arXiv:1302.2394 [hep-ex]].

[47] G. Aad et al. [ATLAS Collaboration], "Rapidity gap cross sections measured with the ATLAS detector in $p p$ collisions at $\sqrt{s}=7$ TeV", Eur. Phys. J. C 72 (2012) 1926 [arXiv:1201.2808 [hep-ex]].

[48] M. Cacciari, G. P. Salam and G. Soyez, "The Anti-k(t) jet clustering algorithm", JHEP 0804 (2008) 063 [arXiv:0802.1189 [hep-ph]].

[49] R. Field, "Early LHC Underlying Event Data - Findings and Surprises" [arXiv:1010.3558 [hep-ph]].

[50] P. Bruni and G. Ingelman, "Diffractive hard scattering at e p and p anti-p colliders", Conf. Proc. C 930722 (1993) 595.

[51] S. Navin, "Diffraction in Pythia" [arXiv:1005.3894 [hep-ph]].

[52] A. Hocker and V. Kartvelishvili, "SVD approach to data unfolding", Nucl. Instrum. Meth. A 372 (1996) 469 [arXiv:9509307 [hep-ph]].

[53] G. D'Agostini, "A Multidimensional unfolding method based on Bayes' theorem", Nucl. Instrum. Meth. A 362 (1995) 487.

[54] B. E. Cox and J. R. Forshaw, "POMWIG: HERWIG for diffractive interactions", Comput. Phys. Commun. 144 (2002) 104 [arXiv:0010303 [hep-ph]].

[55] S. Alioli, K. Hamilton, P. Nason, C. Oleari and E. Re, "Jet pair production in POWHEG", JHEP 1104 (2011) 081 [arXiv:1012.3380 [hep-ph]].

[56] G. Corcella, I. G. Knowles, G. Marchesini, S. Moretti, K. Odagiri, P. Richardson, M. H. Seymour and B. R. Webber, "HERWIG 6: An Event generator for hadron emission reactions with interfering gluons (including supersymmetric processes)", JHEP 0101 (2001) 010 [arXiv:0011363 [hep-ph]].

[57] V. Khachatryan et al. [CMS Collaboration], "Measurements of Inclusive $W$ and $Z$ Cross Sections in $p p$ Collisions at $\sqrt{s}=7$ TeV", JHEP 1101 (2011) 080 [arXiv:1012.2466 [hepex]].

[58] A. Buckley, H. Hoeth, H. Lacker, H. Schulz and J. E. von Seggern, "Systematic event generator tuning for the LHC", Eur. Phys. J. C 65 (2010) 331 [arXiv:0907.2973 [hep-ph]].

[59] M. Bahr, S. Gieseke, M. A. Gigg, D. Grellscheid, K. Hamilton, O. Latunde-Dada, S. Platzer and P. Richardson et al., "Herwig++ Physics and Manual", Eur. Phys. J. C 58 (2008) 639 [arXiv:0803.0883 [hep-ph]]. 
[60] S. Gieseke, C. Rohr and A. Siodmok, "Colour reconnections in Herwig++", Eur. Phys. J. C 72 (2012) 2225 [arXiv:1206.0041 [hep-ph]].

[61] K. Werner, F. M. Liu and T. Pierog, "Parton ladder splitting and the rapidity dependence of transverse momentum spectra in deuteron-gold collisions at RHIC", Phys. Rev. C 74 (2006) 044902 [arXiv:0506232 [hep-ph]].

[62] T. Pierog, I. Karpenko, J. M. Katzy, E. Yatsenko and K. Werner, "EPOS LHC : test of collective hadronization with LHC data" [arXiv:1306.0121 [hep-ph]].

[63] S. Ostapchenko, "Monte Carlo treatment of hadronic interactions in enhanced Pomeron scheme: I. QGSJET-II model", Phys. Rev. D 83 (2011) 014018 [arXiv:1010.1869 [hep-ph]].

[64] T. Pierog, "LHC Results and High Energy Cosmic Ray Interaction Models", J. Phys.: Conf. Ser. 409 (2013) 012008, doi:10.1088/1742-6596/409/1/012008.

[65] S. Chatrchyan et al. [CMS and TOTEM Collaborations], "CMS-TOTEM Precision Proton Spectrometer Technical Design Report", CMS-TDR-13, TOTEM-TDR-003, CERNLHCC-2014-021, September 8, 2014.

[66] TOTEM Collaboration, "Timing Measurements in the Vertical Roman Pots of the TOTEM Experiment", CERN-LHCC-2014-020. TOTEM-TDR-002, September, 2014. 